\title{
High-Risk Screening for Fabry Disease: A Nationwide Study in Japan and Literature Review
}

\author{
Takaaki Sawada ${ }^{1,2}$, Jun Kido ${ }^{1, * \mathbb{C}}$, Keishin Sugawara $^{1}$ and Kimitoshi Nakamura ${ }^{1} \mathbb{C}$ \\ 1 Department of Pediatrics, Faculty of Life Sciences, Kumamoto University, 1-1-1 Honjo, \\ Kumamoto City 860-8556, Japan; sawada.takaki@kuh.kumamoto-u.ac.jp (T.S.); \\ sugawara.kei2552@gmail.com (K.S.); nakamura@kumamoto-u.ac.jp (K.N.) \\ 2 Department of Pediatrics, Graduate School of Medical Sciences, Kumamoto University, \\ Kumamoto City 860-8556, Japan \\ * Correspondence: kidojun@kuh.kumamoto-u.ac.jp; Tel.: +81-096-373-5191; Fax: +81-096-373-5335
}

check for updates

Citation: Sawada, T.; Kido, J.; Sugawara, K.; Nakamura, K. High-Risk Screening for Fabry Disease: A Nationwide Study in Japan and Literature Review. Diagnostics 2021, 11, 1779. https:// doi.org/10.3390/diagnostics11101779

Academic Editor: Ivana Kholová

Received: 31 August 2021

Accepted: 24 September 2021

Published: 27 September 2021

Publisher's Note: MDPI stays neutral with regard to jurisdictional claims in published maps and institutional affiliations.

Copyright: (c) 2021 by the authors. Licensee MDPI, Basel, Switzerland. This article is an open access article distributed under the terms and conditions of the Creative Commons Attribution (CC BY) license (https:// creativecommons.org/licenses/by/ $4.0 /)$.

\begin{abstract}
Fabry disease (FD) is an X-linked inherited disorder caused by mutations in the GLA gene, which encodes the lysosomal enzyme $\alpha$-galactosidase A ( $\alpha$-Gal A). FD detection in patients at an early stage is essential to achieve sufficient treatment effects, and high-risk screening may be effective. Here, we performed high-risk screening for FD in Japan and showed that peripheral neurological manifestations are important in young patients with FD. Moreover, we reviewed the literature on high-risk screening in patients with renal, cardiac, and central neurological manifestations. Based on the results of this study and review of research abroad, we believe that FD can be detected more effectively by targeting individuals based on age. In recent years, the methods for highrisk screening have been ameliorated, and high-risk screening studies using GLA next-generation sequencing have been conducted. Considering the cost-effectiveness of screening, GLA sequencing should be performed in individuals with reduced $\alpha$-Gal A activity and females with certain FD manifestations and/or a family history of FD. The findings suggest that family analysis would likely detect FD patients, although GLA sequencing of asymptomatic family members requires adequate genetic counseling.
\end{abstract}

Keywords: $\alpha$-galactosidase A; Fabry disease; GLA; high-risk screening; newborn screening

\section{Introduction}

Fabry disease (FD; MIM 301500) is an X-linked inherited disorder caused by mutations in the GLA gene and resulting in the impaired activity of the lysosomal enzyme $\alpha$ galactosidase A ( $\alpha$-Gal A; EC 3.2.1.22) [1]. $\alpha$-Gal A deficiency causes globotriaosylceramide (Gb3) accumulation in cells throughout the body, leading to various clinical manifestations. Moreover, globotriaosylsphingosine (Lyso-Gb3), which is the deacylated form of Gb3, is cytotoxic and exerts inflammation-inducing and fibrosis-promoting effects [2].

Based on the clinical manifestations and onset age, men with FD are classified into two types: the classical type, where patients develop symptoms, such as limb pain, acroparesthesia, sweating disorders, angiokeratoma, and/or gastrointestinal symptoms in childhood or adolescence; and the late-onset type, where patients develop kidney, heart, and cerebrovascular disorders in adulthood or at an older age. In addition, because FD is an X-linked inheritance, some females can develop these manifestations, and heterozygous female patients develop symptoms similar to those of the classical type, suggesting the involvement of lyonization [3].

Enzyme replacement therapy (ERT) is a currently available treatment option for FD that slows renal deterioration, alleviates the progression of cardiomyopathy, and prevents morbidity and death [4]. Moreover, a pharmacological chaperone therapy (Migalastat; 1-deoxygalactonojirimycin) was recently approved for FD patients with amenable GLA variants [5]. Migalastat that selectively and reversibly binds to the active sites of amenable 
mutant forms of $\alpha$-Gal A enzyme [6,7]. Given its efficacy, convenient oral regimen and the limited therapeutic options available, migalastat can be an important therapeutic option for FD patients with migalastat-amenable GLA variants [8]. Responsiveness of each variant should be carefully evaluated [9-11]. Early treatment is critical to preserve organ function; however, many patients are diagnosed at later stages or misdiagnosed owing to the nonspecific clinical manifestations of the early stages of the disease [12,13]. Newborn screening (NBS), which is effective for early detection of patients with FD, revealed that FD is more frequent than expected in Japan and worldwide [14-18], suggesting that there might be many undiagnosed patients with FD. High-risk screening is one method of FD detection via uniform screening in patients with FD-related symptoms, including renal, cardiac, and central neurological manifestations. Various high-risk screening programs are performed worldwide using different target populations and screening methods. In Japan, we previously performed high-risk screening in patients with risk factors for FD [13], and gene and substrate-reducing therapies for FD are currently undergoing clinical trials worldwide [19]. The approval of these new treatments will make high-risk screening for FD increasingly important because patients with FD can be treated using these new medicines. Here, we review the outcomes of the high-risk screening performed in our study and those worldwide and discuss future methods of high-risk screening.

\section{Targets for High-Risk Screening of FD}

The clinical course of FD varies depending on the disease type. The classical type, which includes some heterozygous females, shows symptoms, such as limb pain, acroparesthesia, sweating disorders, and/or gastrointestinal symptoms in childhood, with cardiac, renal, and cerebrovascular diseases appearing in adulthood and older people [20]. However, in most cases, limb pain or acroparesthesia disappears in adulthood and older people [21]. The late-onset type develops cardiac, renal, and/or cerebrovascular diseases after adolescence and without classical manifestations [22]. In some NBS, late-onset variants are detected more often than classical variants [14,15]. Additionally, patients with late-onset FD might be more common than those with classical FD; therefore, high-risk screening is important for individuals with FD-related manifestations and who do not receive NBS. Most high-risk screening targets patients with cardiac, renal, or cerebrovascular diseases. Specifically, those presenting left ventricular hypertrophy (LVH) or hypertrophic cardiomyopathy $(\mathrm{HCM})$ related to cardiac disease, undergoing dialysis and/or kidney transplants for renal disease, and younger patients with stroke-related cerebrovascular disease.

\section{Methods for High-Risk Screening}

There are several methods for FD screening, including measuring $\alpha$-Gal A enzyme activity in the blood, Gb3 level in urine or blood and/or Lyso-Gb3 level in the blood, and GLA gene sequencing.

$\alpha-G a l$ A, which is extracted from dried blood spots (DBSs), plasma, or serum, reacts with artificial substrates, and its activity is measured by a fluorescence plate reader or tandem mass spectrometry [23]. DBS is useful for NBS because it is easy to collect, store, and transport and can be used to evaluate those of individuals simultaneously. However, it should be noted that decreases in $\alpha$-Gal A activity might not be detectable in female patients with FD, even when using each assay method [24].

Moreover, it is possible that methods for measuring Gb3 accumulation in urine and blood [25,26] and Lyso-Gb3 in blood [27] might be insufficient for some female patients with FD [28]. Individuals are likely to have false-positive results when they harbor nonpathogenic polymorphisms. To prevent these false positives, high-risk screening using GLA sequencing should be performed. However, depending on the region of the GLA gene to be analyzed, there are some gene variants that cannot be detected. For example, deep intronic variants, such as IVS4+919G >A, are not detected during the analysis of exons and/or intron regions close to exons [29]. Conversely, analysis of the whole GLA gene might identify 
multiple variants of unknown significance (VOUS), including intronic variants, that are difficult to interpret.

\section{GLA Polymorphisms}

Some variants result in amino acid substitutions and/or low enzyme activity but do not present FD-related manifestations. The p.E66Q, p.D313Y, p.A143T, p.S126G, and p.R118C variants are not regarded as pathogenic [30-34], and evaluation of these variants as pathogenic is controversial [35-37]. Therefore, we considered these five variants as polymorphisms without pathogenicity and subsequently calculated the frequency of patients with FD, excluding these five variants.

\section{Algorithms for Screening and Diagnosis}

For high-risk screening, individuals meeting the selection criteria receive information about FD and the form for informed consent. Possibly, screening tests might not have previously been undertaken in order to reduce the false-positive rate. In our high-risk screening algorithm, individuals showing low DBS $\alpha$-Gal A activity (<a cut-off) in the two times tests (first and second assays) underwent GLA gene sequencing. Detection of a known pathogenic variant by GLA sequencing results in a definitive FD diagnosis; however, the detection of a novel variant or VOUS results in a diagnosis made comprehensively based on the clinical course, family history, $\alpha$-Gal A activity level, Lyso-Gb3 level in the blood, and histological findings. Individuals with positive results receive a definitive diagnosis.

\section{High-Risk Screening in Japan}

We had conducted NBS for FD in western Japan and reported that one in 7683 infants might develop FD [14]. This frequency is similar to that of NBS in the United States [18,38,39]. To investigate the effect of high-risk screening for individuals with FD-related manifestations not receiving NBS, we conducted high-risk screening using the NBS method for FD [13]. The outcome of the high-risk screening in Japan is summarized below.

We screened 18,135 individuals throughout Japan with at least one of the following five risk factors: renal manifestations (e.g., proteinuria, chronic kidney disease, diabetic nephropathy, mulberries in urine, and/or receiving dialysis), cardiac manifestations (e.g., LVH on electrocardiography or echocardiography), central neurological manifestations (e.g., parkinsonism, hearing loss, and/or history of stroke), peripheral neurological manifestations (e.g., limb pain and acroparesthesia), or family history of FD. In individuals with a family history of FD, some of their relatives within the third degree of kinship had already been diagnosed with FD. Individuals who screened positive according to $\alpha$-Gal A activity in DBS underwent GLA sequencing for diagnosis. Twenty-eight individuals $(0.4 \%, 28 / 8823)$ with renal manifestations, $29(0.9 \%, 29 / 4057)$ with cardiac manifestations, and five $(0.2 \%, 5 / 3075)$ with central neurological manifestations were diagnosed with FD. FD was mostly detected in individuals with peripheral neurological manifestations $(4.4 \%$, 30/894), which are considered important risk factors for early detection of FD. Among other risk-factor groups, FD was diagnosed in 128 of $715(23.4 \%)$ individuals with a family history of FD, and in four of 571 (1.7\%) individuals screened for other reasons.

The most frequent variant was p.M296I, which was found in five families. In our screening, these probands were all found in individuals with renal manifestations, although some patients also developed cardiac manifestations [40]. This variant is considered a lateonset variant [41]. The next most common variant was p.R112C, which was found in four families and associated with cardiac symptoms, is considered a classical variant [42]. Seven variants were found in three families: p.R220*, p.R227*, p.K240Efs*8, p.S345Rfs*28, and p.T412Sfs $* 37$ are considered classical variants; and, p.R301Q and p.R112H are considered late-onset variants [42-45]. Therefore, our high-risk screening effectively detected classical and late-onset FD, as well as heterozygous female patients with FD in the Japanese population. 


\section{High-Risk Screening Programs for FD}

High-risk screening for FD has been performed in different areas worldwide. We conducted a review of the literature on high-risk screening for FD in individuals with renal, cardiac, and central neurological manifestations, with studies lacking a description of detected variants excluded.

\subsection{High-Risk Screening for FD in Individuals with Renal Manifestations}

We reviewed 34 high-risk screening studies for FD in individuals with renal manifestations (Table 1). Twenty-nine studies screened individuals with end-stage renal failure and those receiving dialysis and/or kidney transplants [46-73]. Four studies screened individuals with chronic kidney disease before end-stage renal failure [74-78], and one study screened individuals with reduced estimated glomerular filtration rate (eGFR) [79]. In this manifestation category, large-scale screening studies were conducted because more subjects met the selection criteria.

Table 1. Previous high-risk screening for FD in individuals with renal manifestations.

\begin{tabular}{|c|c|c|c|c|c|}
\hline $\begin{array}{c}\text { Criteria } \\
\text { [Patients Screened }(\mathrm{M} \mid \mathrm{F}), n]\end{array}$ & Age Range & $\begin{array}{c}\text { Primary Screening } \\
\text { Test }\end{array}$ & $\begin{array}{c}\text { Patients with Variants } \\
\text { (M|F), } n \\
\text { [Prevalence (M|F), \%]† }\end{array}$ & Detected Variants ( $n$ ) & $\begin{array}{l}\text { Reference, Year, } \\
\text { Country }\end{array}$ \\
\hline $\begin{array}{l}\text { Dialysis patients } \\
{[722(440 \mid 282)]}\end{array}$ & unknown & Plasma $\alpha-\mathrm{Gal} A$ & $\begin{array}{c}2(2 \mid 0) \\
{[0.3(0.5 \mid 0)]}\end{array}$ & p.Q357* (1) & $\begin{array}{l}\text { Utsumi [46], } \\
\text { 2000, Japan }\end{array}$ \\
\hline $\begin{array}{l}\text { Dialysis patients } \\
{[514(514 \mid-)]}\end{array}$ & $20-90$ & Plasma $\alpha-$ Gal A & $\begin{array}{l}5(5 \mid-) \\
{[1.0(1.0 \mid-)]}\end{array}$ & $\begin{array}{c}\text { p.M296I (3), p.A97V (1), p.G373D (1), } \\
\text { p.E66Q (1)\# }\end{array}$ & $\begin{array}{l}\text { Nakao [47], } \\
\text { 2003, Japan }\end{array}$ \\
\hline $\begin{array}{l}\text { Dialysis patients } \\
\text { [2480 (1516 | 964)] }\end{array}$ & 61.8 (mean) & DBS $\alpha-G a l$ A & $\begin{array}{c}4(4 \mid 0) \\
{[0.2(0.3 \mid 0)]}\end{array}$ & $\begin{array}{c}\text { p.A121P(1), p.W162R (1), p.I239T (1), } \\
\text { p.R112H (1) }\end{array}$ & $\begin{array}{l}\text { Kotanko [50], } \\
\text { 2004, Austria }\end{array}$ \\
\hline $\begin{array}{l}\text { Dialysis patients } \\
\text { [450(450|-)] }\end{array}$ & $26-89$ & Plasma $\alpha-$ Gal A & $\begin{array}{c}1(1 \mid-) \\
{[0.2(0.2 \mid-)]}\end{array}$ & g.10252_10254del3 (1) & $\begin{array}{l}\text { Ichinose [60], } \\
\text { 2005, Japan }\end{array}$ \\
\hline $\begin{array}{l}\text { Dialysis patients } \\
{[3370(1521 \mid 1849)]}\end{array}$ & unknown & DBS $\alpha-G a l$ A & $\begin{array}{c}4(3 \mid 1) \\
{[0.1(0.2 \mid 0.1)]}\end{array}$ & $\begin{array}{c}\text { p.G360S (1), p.R112H (1), p.I317T (1), } \\
\text { p.Q280K (1), p.A143T (1)\# }\end{array}$ & $\begin{array}{l}\text { Merta [61], 2007, } \\
\text { Czech Republic }\end{array}$ \\
\hline $\begin{array}{l}\text { Dialysis patients } \\
\text { [922 }(180 \mid 742)]\end{array}$ & $\begin{array}{l}18-80 \text { (males) } \\
>16 \text { (females) }\end{array}$ & DBS $\alpha-G a l$ A & $\begin{array}{c}1(1 \mid 0) \\
{[0.1(0.6 \mid 0)]}\end{array}$ & p.W236R (1), p.A143T (2)\# & $\begin{array}{l}\text { Terryn [62], } \\
\text { 2007, Belgium }\end{array}$ \\
\hline $\begin{array}{c}\text { CKD patients involving } \\
\text { dialysis and } \\
\text { transplant patients } \\
{[499(499 \mid-)]}\end{array}$ & 63 (mean) & Plasma $\alpha-G a l$ A & $\begin{array}{c}0(0 \mid-) \\
{[0(0 \mid-)]}\end{array}$ & & $\begin{array}{l}\text { Andrade [74], } \\
\text { 2008, Canada }\end{array}$ \\
\hline $\begin{array}{l}\text { Dialysis patients } \\
{[480(311 \mid 169)]}\end{array}$ & $\begin{array}{l}>40 \text { (male) } \\
>50 \text { (female) }\end{array}$ & Serum Gb3 & $\begin{array}{c}0(0 \mid 0) \\
{[0(0 \mid 0)]}\end{array}$ & & $\begin{array}{l}\text { Kim [63], } \\
\text { 2010, Korea }\end{array}$ \\
\hline $\begin{array}{l}\text { Dialysis patients } \\
{[911(543 \mid 368)]}\end{array}$ & $20-91$ & DBS $\alpha-$ Gal A & $\begin{array}{c}2(1 \mid 1) \\
{[0.2(0.2 \mid 0.3)]}\end{array}$ & $\begin{array}{l}\text { c.595_596insG (1), c.1037delG (1), } \\
\text { p.R118C (3)\#, p.D313Y (2)\# }\end{array}$ & $\begin{array}{l}\text { Gaspar [64], } \\
\text { 2010, Spain }\end{array}$ \\
\hline $\begin{array}{l}\text { Dialysis patients } \\
{[933(557 \mid 376)]}\end{array}$ & unknown & DBS $\alpha-G a l$ A & $\begin{array}{c}1(0 \mid 1) \\
{[0.1(0 \mid 0.3)]}\end{array}$ & p.A73E (1), p.E66Q (2)\# & $\begin{array}{l}\text { Nishino, et al. } \\
\text { [65], 2012, Japan }\end{array}$ \\
\hline $\begin{array}{l}\text { Dialysis patients } \\
{[1080(1080 \mid-)]}\end{array}$ & 63.4 (mean) & Plasma $\alpha-G a l$ A & $\begin{array}{c}2(2 \mid-) \\
{[0.2(0.2 \mid-)]}\end{array}$ & p.G195V (1), p.M296I (1), p.E66Q (8)\# & $\begin{array}{l}\text { Doi [66], } \\
\text { 2012, Japan }\end{array}$ \\
\hline Dialysis patients & $18-90$ & DBS $\alpha-G a l$ A & $\begin{array}{l}2(2 \mid 0) \\
0.2(0.3 \mid 0)]\end{array}$ & p.L275F (1), p.P214S (1) & $\begin{array}{l}\text { Okur [58], } \\
\text { 2013, Turkey }\end{array}$ \\
\hline $\begin{array}{c}\text { Dialysis patients } \\
{[1453(1453 \mid-)]}\end{array}$ & $25-95$ & $\begin{array}{l}\text { Plasma } \alpha-G a l \text { A, } \\
\text { Plasma Lyso-Gb3 }\end{array}$ & $\begin{array}{c}1(1 \mid-) \\
{[0.1(0.1 \mid-)]}\end{array}$ & p.Y173* (1), p.E66Q (9)\# & $\begin{array}{l}\text { Maruyama [67], } \\
\text { 2013, Japan }\end{array}$ \\
\hline $\begin{array}{l}\text { Dialysis patients } \\
{[1401(1401 \mid-)]}\end{array}$ & unknown & DBS $\alpha-$ Gal A & $\begin{array}{c}2(2 \mid-) \\
{[0.1(0.1 \mid-)]}\end{array}$ & p.R363H (1), p.R112H (1) & $\begin{array}{l}\text { Rozenfeld [68], } \\
\text { 2015, Argentina }\end{array}$ \\
\hline $\begin{array}{l}\text { CKD stage1-5 } \\
{[313(167 \mid 146)]}\end{array}$ & $18-70$ & DBS $\alpha-G a l$ A & $\begin{array}{c}3(3 \mid 0) \\
{[1.0(1.8 \mid 0)]}\end{array}$ & $\begin{array}{c}\text { p.N34H (1), c.1072_1074del(p.358delE) } \\
\text { (1), p.F229V (1) }\end{array}$ & $\begin{array}{l}\text { Turkmen [75], } \\
\text { 2016, Turkey }\end{array}$ \\
\hline $\begin{array}{l}\text { Dialysis patients } \\
{[8547(5408 \text { | } 3139)]}\end{array}$ & $5-98$ & Plasma $\alpha-$ Gal A & $\begin{array}{c}2(2 \mid 0) \\
{[0.02(0.04 \mid 0)]}\end{array}$ & p.R112C (2), p.E66Q (11)\# & $\begin{array}{l}\text { Saito [69], } \\
\text { 2016, Japan }\end{array}$ \\
\hline $\begin{array}{l}\text { Dialysis patients } \\
{[1527(847 \mid 680)]}\end{array}$ & 60.2 (mean) & DBS $\alpha-G a l$ A & $\begin{array}{c}4(4 \mid 0) \\
{[0.3(0.5 \mid 0)]}\end{array}$ & $\begin{array}{l}\text { p.M296V (1), p.R112H (1), p.S65N (1), } \\
\text { c.1212_1214delAAG (1), p.D313Y (1)\# }\end{array}$ & $\begin{array}{l}\text { Sayilar [70], } \\
\text { 2016, Turkey }\end{array}$ \\
\hline $\begin{array}{l}\text { Dialysis patients } \\
{[2.583(2583 \mid-)]}\end{array}$ & $18-91$ & DBS $\alpha-G a l$ A & $\begin{array}{c}3(3 \mid-) \\
{[0.1(0.1 \mid-)]}\end{array}$ & p.W204* (1), p.A368T (1), p.C52F (1) & $\begin{array}{l}\text { Silva [71], } \\
\text { 2016, Brazil }\end{array}$ \\
\hline $\begin{array}{l}\text { Dialysis patients } \\
\text { [142 (81 | 61)] }\end{array}$ & $20-60$ & DBS $\alpha-G a l$ A & $\begin{array}{c}0(0 \mid 0) \\
{[0(0 \mid 0)]}\end{array}$ & & $\begin{array}{l}\text { Trachoo [72], } \\
\text { 2017, Thailand }\end{array}$ \\
\hline $\begin{array}{l}\text { CKD not on dialysis } \\
{[1453(783 \mid 656)]}\end{array}$ & 59.3 (mean) & DBS $\alpha-$-Gal A & $\begin{array}{c}0(0 \mid 0) \\
{[0(0 \mid 0)]}\end{array}$ & p.D313Y (2)\#, p.A143T (1)\# & $\begin{array}{l}\text { Yeniçerioğlu [76], } \\
\text { 2017, Turkey }\end{array}$ \\
\hline $\begin{array}{c}\text { Kidney transplant recipients } \\
{[1095(648 \mid 447)]}\end{array}$ & unknown & $\begin{array}{c}\text { DBS } \alpha \text {-Gal A } \\
\text { (male), GLA } \\
\text { sequencing } \\
\text { (female) }\end{array}$ & $\begin{array}{c}1(1 \mid 0) \\
{[0.1(0.2 \mid 0)]}\end{array}$ & p.Q330R (1), p.D313Y (2)\#, p.S126G (1)\# & $\begin{array}{l}\text { Y1lmaz [73], } \\
\text { 2017, Turkey }\end{array}$ \\
\hline $\begin{array}{l}\text { Dialysis patients } \\
\text { [108 (108|-)] }\end{array}$ & unknown & DBS $\alpha-$ Gal A & $\begin{array}{c}1(1 \mid-) \\
{[0.9(0.9 \mid-)]}\end{array}$ & p.G35V (1) & $\begin{array}{l}\text { Veloso [48], } \\
\text { 2018, Brazil }\end{array}$ \\
\hline $\begin{array}{l}\text { Dialysis patients } \\
{[227(148 \mid 79)]}\end{array}$ & 65 (mean) & GLA sequencing & $\begin{array}{c}1(1 \mid 0) \\
{[0.4(0.7 \mid 0)]}\end{array}$ & p.I91T (1), p.D313Y (1)\# & $\begin{array}{l}\text { Zizzo [49], } \\
\text { 2018, Italy }\end{array}$ \\
\hline $\begin{array}{c}\text { Pre-end stage renal disease } \\
{[1012(1012 \mid-)]}\end{array}$ & $20-85$ & DBS $\alpha-$ Gal A & $\begin{array}{c}6(6 \mid-) \\
{[0.6(0.6 \mid-)]}\end{array}$ & $\begin{array}{c}\text { p.T410A (1), p.G318E (1), IVS4+919G >A } \\
\text { (3), p.P210S }\end{array}$ & $\begin{array}{l}\text { Lin [77], } \\
\text { 2018, Taiwan }\end{array}$ \\
\hline
\end{tabular}


Table 1. Cont.

\begin{tabular}{|c|c|c|c|c|c|}
\hline $\begin{array}{c}\text { Criteria } \\
{[\text { Patients Screened (M|F), } n]}\end{array}$ & Age Range & $\begin{array}{c}\text { Primary Screening } \\
\text { Test }\end{array}$ & $\begin{array}{c}\text { Patients with Variants } \\
(\mathrm{M} \mid \mathrm{F}), n \\
\text { [Prevalence (M|F), \%]† }\end{array}$ & Detected Variants $(n)$ & $\begin{array}{l}\text { Reference, Year, } \\
\text { Country }\end{array}$ \\
\hline $\begin{array}{l}\text { Dialysis patients } \\
\text { [9604 (9604|-)] }\end{array}$ & $18-100$ & DBS $\alpha-G a l$ A & $\begin{array}{c}24(24 \mid-) \\
{[0.2(0.2 \mid-)]}\end{array}$ & $\begin{array}{c}\text { p.C56S (1), p.L415P (4), c.640-1G>C (1), } \\
\text { p.R363H (3), p.G85D (1), c.886_887delAT } \\
\text { (1), Deletion exons 3-4 (2), } \\
\text { c.1235_1236delCT(p.T412fs) (1), } \\
\text { p.D109G (2), p.W81* (1), p.P205S (1), } \\
\text { p.P409S (1), p.A156_A160del (1), } \\
\text { c.902_905insTGTC (1), p.D55G (1), } \\
\text { p.G80D (1) }\end{array}$ & $\begin{array}{l}\text { Frabasil [51], } \\
\text { 2019, Argentina }\end{array}$ \\
\hline $\begin{array}{l}\text { Dialysis patients } \\
{[5572(3551 \mid 2021)]}\end{array}$ & $18-59$ & DBS $\alpha-G a l$ A & $\begin{array}{c}20(19 \mid 1) \\
{[0.4(0.5 \mid 0.05)]}\end{array}$ & $\begin{array}{l}\text { p.F273S (1), p.L54P (3), p.N215S (1), } \\
\text { p.Y134D (1), p.W262* (1), p.R220* (2), } \\
\text { p.R342Q (1), p.D170Y (1), p.W399* (1), } \\
\text { p.R227* (1), p.W204C (1), p.E7* (1), } \\
\text { p.G183S (1), p.A37S (1), p.C56S (1), } \\
\text { p.L68P (1), p.G328R (1) }\end{array}$ & $\begin{array}{l}\text { Moiseev [52], } \\
\text { 2019, Russia }\end{array}$ \\
\hline $\begin{array}{c}\text { Dialysis patients and } \\
\text { patients with transplant } \\
{[5657(3442 \mid 2215)]}\end{array}$ & 48 (mean) & $\begin{array}{l}\text { DBS } \alpha-\text {-Gal A } \\
\text { (male), GLA } \\
\text { sequencing } \\
\text { (female) }\end{array}$ & $\begin{array}{c}13(9 \mid 4) \\
{[0.2(0.3 \mid 0.2)]}\end{array}$ & $\begin{array}{l}\text { p.R227Q (1), p.Q330R (1), p.V199M (1), } \\
\text { p.P205T (1), p.E59V (1), IVS6-10G >A (1), } \\
\text { p.L54F (1), p.358delE (1), p.S364C (1), } \\
\text { p.T39S (1), p.L8Q (1), p.P205S (1), } \\
\text { p.C223* (1), p.S126G (4)\#, p.D313Y (19)\# }\end{array}$ & $\begin{array}{l}\text { Yalın [53], } \\
\text { 2019, Turkey }\end{array}$ \\
\hline $\begin{array}{l}\text { Dialysis patients } \\
{[526(325 \mid 201)]}\end{array}$ & unknown & DBS $\alpha-G a l$ A & $\begin{array}{c}0(0 \mid 0) \\
{[0(0 \mid 0)]}\end{array}$ & & $\begin{array}{l}\text { Jahan [54], } \\
\text { 2020, Australia }\end{array}$ \\
\hline $\begin{array}{l}\text { Kidney transplant recipients } \\
{[265(175 \mid 90)]}\end{array}$ & 53.6 (mean) & $\begin{array}{l}\text { GLA sequencing, } \\
\text { DBS } \alpha-\text {-Gal A, DBS } \\
\text { Lyso- Gb3 }\end{array}$ & $\begin{array}{c}3(1 \mid 2) \\
{[1.1(0.6 \mid 2.2)]}\end{array}$ & $\begin{array}{l}\text { p.F113L (1), p.D615H (1), p.R220* (1), } \\
\text { p.D313Y (2)\#, p.S126G (2)\# }\end{array}$ & $\begin{array}{l}\text { Veroux [55], } \\
\text { 2020, Italy }\end{array}$ \\
\hline $\begin{array}{l}\text { Kidney transplant recipients } \\
{[301(180 \mid 120)]}\end{array}$ & 43 (mean) & GLA sequencing & $\begin{array}{c}1(1 \mid 0) \\
{[0.3(0.6 \mid 0)]}\end{array}$ & $\begin{array}{l}\text { c.1093_1101dup (1), p.D313Y (1)\#, } \\
\text { p.A143T (1)\# }\end{array}$ & $\begin{array}{l}\text { Erdogmus [56], } \\
\text { 2020, Turkey }\end{array}$ \\
\hline $\begin{array}{l}\text { CKD stage } 3-5 \\
{[397(279 \mid 118)]}\end{array}$ & $32-75$ & $\begin{array}{l}\text { Dried urine spots } \\
\text { Gb3 }\end{array}$ & $\begin{array}{c}0(0 \mid 0) \\
{[0(0 \mid 0)]}\end{array}$ & & $\begin{array}{l}\text { Auray-Blais [78], } \\
\text { 2020, Canada }\end{array}$ \\
\hline $\begin{array}{l}\text { Dialysis patients } \\
\text { [619(unknown)] }\end{array}$ & $>18$ & DBS $\alpha-G a l$ A & $\begin{array}{c}3(0 \mid 3) \\
{[0.5 \text { (unknown) }]}\end{array}$ & p.A352G (3) & $\begin{array}{l}\text { Alhemyadi [57], } \\
\text { 2020, Saudi } \\
\text { Arabia }\end{array}$ \\
\hline $\begin{array}{c}\text { Reduced estimated } \\
\text { glomerular filtration rate } \\
{[1084(505 \mid 579)]}\end{array}$ & $20-70$ & $\begin{array}{l}\text { DBS } \alpha-\text { Gal A } \\
\text { (male), DBS } \alpha-\text { Gal } \\
\text { A and GLA } \\
\text { sequencing } \\
\text { (female) }\end{array}$ & $\begin{array}{c}1(0 \mid 1) \\
{[0.1(0 \mid 0.2)]}\end{array}$ & p.L300F (1) & $\begin{array}{l}\text { Reynolds [79], } \\
\text { 2021, UK }\end{array}$ \\
\hline $\begin{array}{c}\text { Dialysis patients and } \\
\text { patients with transplant } \\
{[819(819 \mid-)]}\end{array}$ & $18-70$ & DBS $\alpha-G a l$ A & $\begin{array}{c}1(1 \mid-) \\
{[0.1(0.1 \mid-)]}\end{array}$ & p.F113L (1) & $\begin{array}{l}\text { Vigneau [59], } \\
\text { 2021, France }\end{array}$ \\
\hline
\end{tabular}

CKD: Chronic kidney disease, DBS: Dried blood spot; t: Excluding polymorphisms; \#: Polymorphisms (p.E66Q, p.D313Y, p.A143T, p.S126G and p.R118C). Mutation nomenclature followed the guidelines established by the Human Genome Variation Society (http: // varnomen.hgvs.org/, accessed on 10 September 2021).

In these studies, pathogenic variants in the GLA gene were detected at a frequency of up to $1.1 \%(0-1.8 \%$ in males and $0-2.2 \%$ in females), with the frequency in half of these studies ranging from 0.1 to $0.3 \%$. Five studies detected no patients with GLA pathogenic variants, except for polymorphisms, and two of these five studies screened using Gb3 measurements [63,78], with the remaining three using $<600$ subjects for screening $[72,74,76]$.

Each large-scale screening study with $>5000$ subjects [51-53] found $>10$ GLA variants. In these studies, the most common variants were p.L415P (classical), p.R363H (late-onset), p.M296I, and p.R112H and found in Argentina [51,68]. Notably, p.M296I has been detected as a late-onset variant in Japan $[47,66]$. In our screening, we also detected p.M296I in four patients with renal manifestations [13]. p.R112H has previously been detected in Argentina [68], the Czech Republic [61], Austria [50], and Turkey [70] and in two patients with renal manifestations in our high-risk screening [13]. Therefore, p.R363H, p.M296I, and p.R112H might be late-onset variants with a tendency toward the development of renal manifestations.

Most high-risk screening in individuals with renal manifestations targeted patients that had undergone dialysis and/or renal transplantation due to end-stage renal failure. Therefore, the renal manifestations in patients detected by screening could not be improved with ERT or chaperone therapy. High-risk screening studies targeting individuals with renal manifestations prior to end-stage renal failure have also detected FD patients [75,77,79]. Screening at an earlier symptomatic stage (e.g., proteinuria, albuminuria, and decreased eGFR) is considered to contribute to improving clinical outcomes in patients based on them receiving effective early medical treatment. The $\alpha$-Gal A activity assay using DBS 
would be effective in investigating a large population of individuals presenting such symptoms. However, screening by measuring $\alpha$-Gal A activity alone might miss female patients, making screening with GLA sequencing essential. Yalın et al. [53] performed GLA sequencing to screen 2215 females with hemodialysis or kidney transplant and diagnosed four $(0.2 \%)$ heterozygous female patients. This frequency did not differ significantly from that obtained by measuring $\alpha$-Gal A activity $(0-0.3 \%)$.

\subsection{High-Risk Screening for FD in Individuals with Cardiac Manifestations}

We reviewed 23 high-risk screenings for FD in individuals with cardiac manifestations (Table 2), with 20 screenings performed for individuals with either LVH or HCM [40,80-98]. The other three screenings were performed in individuals with a variety of cardiac symptoms, including coronary artery disorders, conduction disorders, cardiomyopathy, and valvular disease [99], those with conduction disorders requiring a pacemaker [100], and those with left ventricular noncompaction (LVNC) [101].

Table 2. Previous high-risk screening for FD in individuals with cardiac manifestations.

\begin{tabular}{|c|c|c|c|c|c|}
\hline $\begin{array}{c}\text { Criteria } \\
\text { [Patients Screened } \\
(\mathrm{M} \mid \mathrm{F}), n]\end{array}$ & Age Range & $\begin{array}{c}\text { Primary Screening } \\
\text { Test }\end{array}$ & $\begin{array}{c}\text { Patients with Variants } \\
(\mathbf{M} \mid \mathrm{F}), n \\
\text { [Prevalence }(\mathbf{M} \mid \mathrm{F}), \%]+\end{array}$ & Detected Variants $(n)$ & $\begin{array}{l}\text { Reference, Year, } \\
\text { Country }\end{array}$ \\
\hline $\begin{array}{c}\text { LVH } \\
{[230(230 \mid-)]}\end{array}$ & $16-87$ & Plasma $\alpha$-Gal A & $\begin{array}{c}7(7 \mid-) \\
{[3.0(3.0 \mid-)]}\end{array}$ & p.M296I (1), p.A20P (1) & $\begin{array}{l}\text { Nakao [40], } \\
\text { 1995, Japan }\end{array}$ \\
\hline $\begin{array}{c}\text { HCM } \\
{[153(153 \mid-)]}\end{array}$ & $8-71$ & Plasma $\alpha$-Gal A & $\begin{array}{c}5(5 \mid-) \\
{[3.3(3.3 \mid-)]}\end{array}$ & $\begin{array}{l}\text { p.N215S (3), p.I317T (1), } \\
\text { c.1223del (1), p.D313Y (1)\# }\end{array}$ & $\begin{array}{l}\text { Sachdev [80], } \\
\text { 2002, UK }\end{array}$ \\
\hline $\begin{array}{c}\text { HCM } \\
{[508(328 \mid 108)]}\end{array}$ & 58 (mean) & Plasma $\alpha$-Gal A & $\begin{array}{c}4(3,1) \\
{[0.8(0.9 \mid 0.9)]}\end{array}$ & $\begin{array}{l}\text { p.L89P (1), p.E358del (1), } \\
\text { p.S238N (2), p.A143T (1)\# }\end{array}$ & $\begin{array}{l}\text { Monserrat [83], } \\
\text { 2007, Spain }\end{array}$ \\
\hline $\begin{array}{c}\text { HCM } \\
{[392(278 \mid 114)]}\end{array}$ & $18-79$ & DBS $\alpha-G a l A$ & $\begin{array}{c}4(4,-) \\
{[1.0(1.4 \mid 0)]}\end{array}$ & $\begin{array}{l}\text { p.T162C (1), p.F113L (2), } \\
\text { p.N215S (1) }\end{array}$ & $\begin{array}{l}\text { Hagege [84], } \\
\text { 2011, France }\end{array}$ \\
\hline $\begin{array}{c}\text { HCM } \\
{[1386(885 \mid 501)]}\end{array}$ & 58 (mean) & GLA sequencing & $\begin{array}{c}4(2 \mid 2) \\
{[0.3(0.2 \mid 0.4)]}\end{array}$ & $\begin{array}{l}\text { p.N215S (2), p.D244N (1), } \\
\text { p.T410A (1), p.R118C (1)\#, } \\
\text { p.A143T (2)\# }\end{array}$ & $\begin{array}{l}\text { Elliott [91], 2011, } \\
\text { 13 European } \\
\text { countries }\end{array}$ \\
\hline $\begin{array}{c}\text { LVH } \\
{[738(738 \mid-)]}\end{array}$ & unknown & Serum $\alpha-G a l$ A & $\begin{array}{c}0(0 \mid-) \\
{[0(0 \mid-)]}\end{array}$ & p.E66Q (3)\# & $\begin{array}{l}\text { Mawatari [85], } \\
\text { 2013, Japan }\end{array}$ \\
\hline $\begin{array}{c}\text { LVH } \\
{[540(362 \mid 178)]}\end{array}$ & $19-93$ & $\begin{array}{c}\text { DBS } \alpha \text {-Gal A } \\
\text { (male), GLA } \\
\text { sequencing } \\
\text { (female) }\end{array}$ & $\begin{array}{c}1(1 \mid 0) \\
{[0.2(0.3 \mid 0)]}\end{array}$ & p.A5E (1), p.A143T (4)\# & $\begin{array}{c}\text { Terryn [86], } \\
\text { 2013, Belgium }\end{array}$ \\
\hline $\begin{array}{c}\text { LVH } \\
{[100(100 \mid-)]}\end{array}$ & $33-83$ & DBS $\alpha$-Gal A & $\begin{array}{c}4(4 \mid-) \\
{[4.0(4.0 \mid-)]}\end{array}$ & $\begin{array}{l}\text { intronic splice variants (2), } \\
\text { p.N215S (1), p.Y152H (1) }\end{array}$ & $\begin{array}{l}\text { Palecek [87], 2014, } \\
\text { Czech Republic }\end{array}$ \\
\hline $\begin{array}{c}\text { LVH } \\
{[47(23 \mid 24)]}\end{array}$ & $25-90$ & DBS $\alpha$-Gal A & $\begin{array}{c}1(0 \mid 1) \\
{[2.1(0 \mid 4.2)]}\end{array}$ & p.W262L (1) & $\begin{array}{l}\text { Baptista [88], } \\
\text { 2015, Portugal }\end{array}$ \\
\hline $\begin{array}{c}\text { LVH } \\
\text { [2596 (1689 | 907)] }\end{array}$ & 64 (mean) & Urinary Gb3 & $\begin{array}{c}0(0 \mid 0) \\
{[0(0 \mid 0)]}\end{array}$ & & $\begin{array}{l}\text { Gaggl [89], } \\
\text { 2016, Austria }\end{array}$ \\
\hline $\begin{array}{c}\text { HCM } \\
{[273(169 \mid 104)]}\end{array}$ & 58 (mean) & DBS $\alpha-$ Gal A & $\begin{array}{c}3(1 \mid 2) \\
{[1.1(0.6 \mid 1.9)]}\end{array}$ & $\begin{array}{c}\text { p.W226* }(1), \text { p.N224S }(1) \\
\text { c. } 547+3 A>G(1)\end{array}$ & $\begin{array}{l}\text { Seo [90], } \\
\text { 2016, Korea }\end{array}$ \\
\hline $\begin{array}{c}\text { HCM } \\
{[177 \text { (unknown)] }}\end{array}$ & $15-87$ & Plasma $\alpha$-Gal A & $\begin{array}{c}2 \text { (unknown) } \\
{[1.1 \text { (unknown) }}\end{array}$ & $\begin{array}{c}\text { p.R112L (1), } \\
\text { IVS4+919G>A (1) }\end{array}$ & $\begin{array}{l}\text { Kubo [92], } \\
\text { 2017, Japan }\end{array}$ \\
\hline $\begin{array}{c}\text { Coronary artery } \\
\text { disease, conduction or } \\
\text { rhythm abnormalities, } \\
\text { non-ishemic } \\
\text { cardiomyopathy, and } \\
\text { valvular dysfunction } \\
{[2256(1404 \mid 852)]}\end{array}$ & $19-95$ & $\begin{array}{c}\text { Urinary Gb3, DBS } \\
\alpha \text {-Gal A, GLA } \\
\text { sequencing }\end{array}$ & $\begin{array}{c}1(0 \mid 1) \\
{[0(0 \mid 0.1)]}\end{array}$ & $\begin{array}{l}\text { p.D83N (1), p.D313Y (7)\#, } \\
\text { p.R118C (2)\# }\end{array}$ & $\begin{array}{l}\text { Schiffmann [99], } \\
\text { 2018, USA }\end{array}$ \\
\hline $\begin{array}{c}\text { HCM } \\
{[585(413 \mid 172)]}\end{array}$ & $\begin{array}{l}50 \text { (mean: male), } \\
57 \text { (mean: female) }\end{array}$ & $\begin{array}{c}\text { DBS } \alpha-\text {-Gal A } \\
\text { (male), GLA } \\
\text { sequencing } \\
\text { (female) }\end{array}$ & $\begin{array}{c}2(1 \mid 1) \\
{[0.3(0.2 \mid 0.6)]}\end{array}$ & p.N215S (2) & $\begin{array}{l}\text { Maron [93], } \\
\text { 2018, USA }\end{array}$ \\
\hline $\begin{array}{c}\text { LVH } \\
{[986(986 \mid-)]}\end{array}$ & unknown & Plasma $\alpha-$ Gal A & $\begin{array}{c}3(3 \mid-) \\
{[0.3(0.3 \mid-)]}\end{array}$ & $\begin{array}{l}\text { p.G328R (1), p.R301Q (1), } \\
\text { p.H46R (1), p.E66Q (2)\# }\end{array}$ & $\begin{array}{c}\text { Kim [94], } \\
\text { 2019, Korea }\end{array}$ \\
\hline $\begin{array}{c}\text { LVH } \\
{[277(215 \mid 62)]}\end{array}$ & $25-79$ & $\begin{array}{l}\text { Plasma } \alpha \text {-Gal A, } \\
\text { Plasma Lyso-Gb3 }\end{array}$ & $\begin{array}{c}2(1 \mid 1) \\
{[0.7(0.5 \mid 1.6)]}\end{array}$ & $\begin{array}{l}\text { intronic splice variants (1), } \\
\text { p.R112H (1), p.E66Q (2)\# }\end{array}$ & $\begin{array}{c}\text { Yamashita [95], } \\
\text { 2019, Japan }\end{array}$ \\
\hline $\begin{array}{c}\text { Conduction disordaers } \\
\text { requiring a pacemaker } \\
{[188(124 \mid 64)]}\end{array}$ & 63 (mean) & $\begin{array}{l}\alpha-G \text { al A (male), } \\
\text { GLA sequencing } \\
\text { (female) }\end{array}$ & $\begin{array}{c}0(0 \mid 0) \\
{[0(0 \mid 0)]}\end{array}$ & p.D313Y (1)\# & $\begin{array}{l}\text { Lopez-Sainz [100], } \\
\text { 2019, Spain }\end{array}$ \\
\hline
\end{tabular}


Table 2. Cont.

\begin{tabular}{|c|c|c|c|c|c|}
\hline $\begin{array}{c}\text { Criteria } \\
\text { [Patients Screened } \\
(\mathrm{M} \mid \mathrm{F}), n]\end{array}$ & Age Range & $\begin{array}{c}\text { Primary Screening } \\
\text { Test }\end{array}$ & $\begin{array}{c}\text { Patients with Variants } \\
\text { (M } \mid \mathrm{F}), n \\
\text { [Prevalence }(\mathrm{M} \mid \mathrm{F}), \%] \dagger\end{array}$ & Detected Variants $(n)$ & $\begin{array}{l}\text { Reference, Year, } \\
\text { Country }\end{array}$ \\
\hline $\begin{array}{c}\text { HCM } \\
{[80(53 \mid 27)]}\end{array}$ & $18-65$ & GLA sequencing & $\begin{array}{c}2(2 \mid 0) \\
{[2.5(3.8 \mid 0)]}\end{array}$ & p.R112C (1), p.R301Q (1) & $\begin{array}{l}\text { Barman [96], } \\
\text { 2019, Turkey }\end{array}$ \\
\hline $\begin{array}{c}\text { LVNC } \\
{[78(49 \mid 29)]}\end{array}$ & 47 (mean) & $\begin{array}{c}\text { DBS } \alpha \text {-Gal A } \\
\text { (male), GLA } \\
\text { sequencing } \\
\text { (female) }\end{array}$ & $\begin{array}{c}0(0 \mid 0) \\
{[0(0 \mid 0)]}\end{array}$ & & $\begin{array}{l}\text { Azevedo [101], } \\
\text { 2019, Portugal }\end{array}$ \\
\hline $\begin{array}{c}\text { LVH } \\
{[190(119 \mid 71)]}\end{array}$ & 47.2 (mean) & GLA sequencing & $\begin{array}{c}0(0 \mid 0) \\
{[0(0 \mid 0)]}\end{array}$ & p.A143T (1)\#, p.D313Y (1)\# & $\begin{array}{l}\text { Barman [98], } \\
\text { 2019, Turkey }\end{array}$ \\
\hline $\begin{array}{l}\text { LVH } \\
\text { [266 (167 | 99)] }\end{array}$ & $27-98$ & DBS $\alpha-$ Gal A & $\begin{array}{c}5(5 \mid 0) \\
{[1.9(3.0 \mid 0)]}\end{array}$ & IVS4+919G>A (5) & $\begin{array}{l}\text { Sadasivan [97], } \\
\text { 2020, Canada and } \\
\text { Hong Kong }\end{array}$ \\
\hline $\begin{array}{c}\text { LVH } \\
{[511(332 \mid 179)]}\end{array}$ & $18-75$ & DBS $\alpha-G a l$ A & $\begin{array}{c}1(1 \mid 0) \\
{[0.2(0.3 \mid 0)]}\end{array}$ & p.N215S (1), p.A143T (1)\# & $\begin{array}{l}\text { Fuller [81], } \\
\text { 2020, Australia }\end{array}$ \\
\hline $\begin{array}{c}\text { LVH } \\
{[499(336 \mid 163)]}\end{array}$ & 66.4 (mean) & DBS $\alpha-$ Gal A & $\begin{array}{c}8(8 \mid 0) \\
{[1.6(2.4 \mid 0)]}\end{array}$ & IVS4+919G>A (8) & $\begin{array}{l}\text { Fan [82], 2021, } \\
\text { Hong Kong }\end{array}$ \\
\hline
\end{tabular}

LVH: Left ventricular hypertrophy, HCM: Hypertrophic cardiomyopathy, DBS: Dried blood spot, LVNC: left ventricular noncompaction; t: Excluding polymorphisms; \#: Polymorphisms (p.E66Q, p.D313Y, p.A143T, p.S126G and p.R118C). Mutation nomenclature followed the guidelines established by the Human Genome Variation Society (http:/ / varnomen.hgvs.org/, accessed on 10 September 2021).

The frequency of pathogenic variants in the GLA gene detected in these screenings was up to $3.3 \%$ ( $0-3.8 \%$ in males and $0-4.2 \%$ in females). That of FD was $>1 \%$ in almost $50 \%$ of these screening studies and higher than that observed in screenings of individuals with renal manifestations. Furthermore, five studies had no patients with pathogenic GLA variants. These included screening using urinary Gb3 in individuals with LVH [89], serum $\alpha$-Gal A activity in males with LVH [85], measurement of $\alpha$-Gal A activity and/or GLA sequencing in individuals with conduction disorders requiring a pacemaker [100], measurement of $\alpha$-Gal A activity and/or GLA sequencing in individuals with LVNC [101], and GLA sequencing in individuals with LVH [98].

The most common variant detected in the screening was IVS4+919G $>$ A in Hong Kong and Japan, with a total of 11 individuals carrying this variant [82,92,97]. This variant was frequently detected in NBS in Taiwan and patients with late-onset FD with cardiac manifestations [17]. Additionally, p.N215S was detected in a total of 11 patients in Italy, the United States, the United Kingdom, France, the Czech Republic, and Australia $[80,81,84,87,91,93]$. Moreover, this variant has been frequently detected in patients with late-onset FD with cardiac manifestations [102], with one such patient detected in our high-risk screening in Japan [13]. p.R301Q was detected in two individuals in Korea and Turkey [94,96]. Individuals with this variant present a variety of symptoms, even within the same family [45]. In our high-risk screening, this variant was detected in two individuals with renal manifestations [13].

Overall, high-risk screening in individuals with cardiac manifestations more frequently detected GLA pathogenic variants than in individuals with renal manifestations. This might be due to the higher frequency of variants, such as IVS4+919G >A and p.N215S, which tend to develop cardiac manifestations. Although most screenings in individuals with LVH or HCM excluded individuals with hypertension and valvular disease, Fan et al. [82] detected IVS4+919G $>$ A in individuals with LVH and hypertension or valvular disease and insisted that manifestations, such as hypertension and valvular disease, should not be excluded. Conversely, screening in individuals with manifestations other than LVH or HCM, such as conduction disturbances and LVNC, did not detect many GLA pathogenic variants. Therefore, screening in individuals with cardiac manifestations other than LVH or HCM might not be as efficient as screening for FD.

Most high-risk screening studies on individuals with cardiac manifestations have been performed by measuring $\alpha$-Gal A activity. Screening using urinary Gb3 detected no patients with FD [89], suggesting that urinary Gb3 levels would not be a suitable screening test for cardiac FD [23]. Elliot et al. [91] screened 501 females with HCM using GLA 
sequencing and detected two GLA pathogenic variants with a frequency of $0.4 \%$, which did not differ significantly from that determined by screening females using $\alpha$-Gal A activity $(0-4.2 \%)$.

\subsection{High-Risk Screening for FD in Individuals with Central Neurological Manifestations}

We reviewed 19 studies [103-121] on high-risk screening in individuals with central neurological manifestations (Table 3). Most of these studies reported screening in individuals with idiopathic strokes. A feature of FD-related central neurological manifestations is juvenile stroke; therefore, 16 studies restricted the upper age limit of the target individuals to between 49 and 60 years. The number of screening subjects ranged from 300 to 1000 in $\sim 50 \%$ of the screenings.

Table 3. Previous high-risk screening for FD in individuals with central neurological manifestations.

\begin{tabular}{|c|c|c|c|c|c|}
\hline $\begin{array}{c}\text { Criteria } \\
\text { [Patients Screened }(\mathbf{M} \mid \mathbf{F}), n]\end{array}$ & Age Range & $\begin{array}{c}\text { Primary Screening } \\
\text { Test }\end{array}$ & $\begin{array}{l}\text { Patients with Variants } \\
\quad(\mathrm{M} \mid \mathrm{F}), n \\
\text { [Prevalence }(\mathrm{M} \mid \mathrm{F}), \%] \dagger\end{array}$ & Detected Variants $(n)$ & $\begin{array}{l}\text { Reference, Year, } \\
\text { Country }\end{array}$ \\
\hline $\begin{array}{l}\text { Cryptogenic stroke } \\
{[103(64 \mid 39)]}\end{array}$ & $16-60$ & DBS $\alpha-G a l$ A & $\begin{array}{c}0(0 \mid 0) \\
{[0(0 \mid 0)]}\end{array}$ & & $\begin{array}{l}\text { Brouns [103], } \\
\text { 2007, Belgium }\end{array}$ \\
\hline $\begin{array}{c}\text { First ischemic stroke } \\
{[558(558 \mid-)]}\end{array}$ & $15-49$ & Plasma $\alpha-$ Gal A & $\begin{array}{c}0(0 \mid-) \\
{[0(0 \mid-)]}\end{array}$ & p.143T (1)\#, p.D313Y (1)\# & $\begin{array}{l}\text { Wozniak [104], } \\
\text { 2010, USA }\end{array}$ \\
\hline $\begin{array}{l}\text { Stroke, unexplained white } \\
\text { matter lesions, or } \\
\text { vertebrobasilar } \\
\text { dolichoectasia } \\
{[1000(547 \mid 453)]}\end{array}$ & $18-60$ & $\begin{array}{l}\text { DBS } \alpha-\text { Gal A } \\
\text { (male), GLA } \\
\text { sequencing } \\
\text { (female) }\end{array}$ & $\begin{array}{c}0(0 \mid 0) \\
{[0(0 \mid 0)]}\end{array}$ & $\begin{array}{c}\text { p.A143T (2)\#, p.S126G (1)\#, } \\
\text { p.D313Y (5)\# }\end{array}$ & $\begin{array}{l}\text { Brouns [110], } \\
\text { 2010, Belgium }\end{array}$ \\
\hline $\begin{array}{c}\text { First stroke } \\
{[493(300 \mid 193)]}\end{array}$ & $18-55$ & GLA sequencing & $\begin{array}{c}0(0 \mid 0) \\
{[0(0 \mid 0)]}\end{array}$ & p.R118C (6)\#, p.D313Y (6)\# & $\begin{array}{l}\text { Baptista [114], } \\
\text { 2010, Portugal }\end{array}$ \\
\hline $\begin{array}{l}\text { Stroke or transient } \\
\text { ischemic attack } \\
{[1046(502 \text { | 544)] }}\end{array}$ & 24-103 & GLA sequencing & $\begin{array}{c}0(0 \mid 0) \\
{[0(0 \mid 0)]}\end{array}$ & p.D313Y (5)\# & $\begin{array}{l}\text { Marquardt [115], } \\
\text { 2012, UK }\end{array}$ \\
\hline $\begin{array}{l}\text { Stroke or transient } \\
\text { ischemic attack } \\
{[150(102 \mid 48)]}\end{array}$ & $18-55$ & Serum $\alpha-G a l$ A & $\begin{array}{c}0(0 \mid 0) \\
{[0(0 \mid 0)]}\end{array}$ & & $\begin{array}{l}\text { Sarikaya [106], } \\
\text { 2012, Switzerland }\end{array}$ \\
\hline $\begin{array}{l}\text { Stroke or transient } \\
\text { ischemic attack } \\
{[5023(2962 \mid 2061)]}\end{array}$ & $18-55$ & GLA sequencing & $\begin{array}{l}\text { unknown } \\
\text { [unknown] }\end{array}$ & $\begin{array}{l}\text { p.V315I (1), p.D83N (1), } \\
\text { p.L415F (1), p.S102L (1), } \\
\text { p.E418G (1),p.R118C (1)\#, } \\
\text { p.S126G (3)\#, p.A143T (4)\#, } \\
\text { p.D313Y (4)\# }\end{array}$ & $\begin{array}{l}\text { Rolfs [116], 2013, } \\
15 \text { European } \\
\text { countries }\end{array}$ \\
\hline $\begin{array}{l}\text { Cryptogenic ischemic stroke } \\
{[100(55 \mid 45)]}\end{array}$ & $16-55$ & $\begin{array}{l}\text { GLA sequencing, } \\
\text { Plasma Lyso-Gb3 }\end{array}$ & $\begin{array}{c}1(1 \mid 0) \\
{[1.0(1.8 \mid 0)]}\end{array}$ & intronic splice variants (1) & $\begin{array}{l}\text { Dubuc [121], } \\
\text { 2013, Canada }\end{array}$ \\
\hline $\begin{array}{l}\text { Stroke, transient ischemic } \\
\text { attack, white matter lesions, } \\
\text { or cerebral } \\
\text { venous thrombosis } \\
{[178(73 \mid 105)]}\end{array}$ & $18-55$ & GLA sequencing & $\begin{array}{c}1(1 \mid 0) \\
{[0.6(1.4 \mid 0)]}\end{array}$ & p.R227Q (1), p.D313Y (1)\# & $\begin{array}{l}\text { Fancellu [117], } \\
\text { 2015, Italy }\end{array}$ \\
\hline $\begin{array}{l}\text { Lacunar stroke } \\
{[994(706 \mid 288)]}\end{array}$ & 56.7 (mean) & GLA sequencing & $\begin{array}{c}0(0 \mid 0) \\
{[0(0 \mid 0)]}\end{array}$ & p.R118C (1)\# & $\begin{array}{l}\text { Kilarski [118], } \\
\text { 2015, UK }\end{array}$ \\
\hline $\begin{array}{c}\text { Acute ischemic stroke or } \\
\text { transient ischemic attack } \\
{[108(66 \mid 42)]}\end{array}$ & $18-60$ & $\begin{array}{c}\text { Leukocyte } \alpha-\text {-Gal A } \\
\text { (male), GLA } \\
\text { sequencing } \\
\text { (female) }\end{array}$ & $\begin{array}{c}3(1 \mid 2) \\
{[2.7(1.5 \mid 4.8)]}\end{array}$ & $\begin{array}{l}\text { p.R301G (1), p.L415R (1), } \\
\text { c.548-3_553del (1) }\end{array}$ & $\begin{array}{l}\text { Romani [111], } \\
\text { 2015, Italy }\end{array}$ \\
\hline $\begin{array}{c}\text { Stroke } \\
{[588(363 \mid 225)]}\end{array}$ & 74.1 (mean) & DBS $\alpha-$ Gal A & $\begin{array}{c}1(0 \mid 1) \\
{[0.2(0 \mid 0.4)]}\end{array}$ & p.M1T (1), p.E66Q (7)\# & $\underset{2017}{\text { Nagamatsu [107], }}$ \\
\hline $\begin{array}{c}\text { Cryptogenic stroke } \\
{[54(30 \mid 24)]}\end{array}$ & $24-55$ & DBS $\alpha-G a l$ A & $\begin{array}{l}2(2 \mid 0) \\
{[3.7(6.7 \mid 0)]}\end{array}$ & p.R227Q (2) & $\begin{array}{l}\text { Gündoğdu [108], } \\
\text { 2017, Yurkey }\end{array}$ \\
\hline $\begin{array}{c}\text { Cryptogenic ischemic stroke } \\
\text { or transient ischemic attack } \\
{[397(218 \mid 179)]}\end{array}$ & $18-55$ & GLA sequencing & $\begin{array}{c}0(0 \mid 0) \\
{[0(0 \mid 0)]}\end{array}$ & p.R118C (1)\#, p.D313Y (1)\# & $\begin{array}{l}\text { Lanthier [119], } \\
\text { 2017, Canada }\end{array}$ \\
\hline $\begin{array}{c}\text { Stroke or transient } \\
\text { ischemic attack } \\
{[311(168 \mid 143)]}\end{array}$ & $18-55$ & $\begin{array}{l}\text { DBS } \alpha-\text { Gal A } \\
\text { (male), GLA } \\
\text { sequencing } \\
\text { (female) }\end{array}$ & $\begin{array}{c}1(0 \mid 1) \\
{[0.3(0 \mid 0.7)]}\end{array}$ & $\begin{array}{c}\text { p.M296I (1), p.S126G (1)\#, } \\
\text { p.D313Y (2)\# }\end{array}$ & $\begin{array}{l}\text { Reisin [112], } \\
\text { 2018, Argentina }\end{array}$ \\
\hline $\begin{array}{c}\text { Ischemic stroke or } \\
\text { intracerebral hemorrhage } \\
{[516(396 \mid 120)]}\end{array}$ & $21-60$ & $\begin{array}{l}\text { Plasma } \alpha-G a l \text { A, } \\
\text { Plasma Lyso-Gb3 }\end{array}$ & $\begin{array}{c}0(0 \mid 0) \\
{[0(0 \mid 0)]}\end{array}$ & p.E66Q (2)\# & $\begin{array}{l}\text { Kinoshita [105], } \\
\text { 2018, Japan }\end{array}$ \\
\hline
\end{tabular}


Table 3. Cont.

\begin{tabular}{|c|c|c|c|c|c|}
\hline $\begin{array}{c}\text { Criteria } \\
\text { [Patients Screened }(\mathrm{M} \mid \mathrm{F}), n]\end{array}$ & Age Range & $\begin{array}{c}\text { Primary Screening } \\
\text { Test }\end{array}$ & $\begin{array}{c}\text { Patients with Variants } \\
(\mathbf{M} \mid \mathbf{F}), n \\
\text { [Prevalence }(\mathbf{M} \mid \mathrm{F}), \%]+\end{array}$ & Detected Variants $(n)$ & $\begin{array}{l}\text { Reference, Year, } \\
\text { Country }\end{array}$ \\
\hline $\begin{array}{c}\text { Ischemic stroke or } \\
\text { intracerebral hemorrhage } \\
{[1000(750 \mid 250)]}\end{array}$ & $18-55$ & $\begin{array}{l}\text { GLA sequencing } \\
\text { (26 variants) }\end{array}$ & $\begin{array}{c}2(2 \mid 0) \\
{[0.2(0.3 \mid 0)]}\end{array}$ & IVS4+919G>A (2) & $\begin{array}{l}\text { Lee [120], } \\
\text { 2019, Taiwan }\end{array}$ \\
\hline $\begin{array}{l}\text { Cryotogenic stroke } \\
{[50(27 \mid 23)]}\end{array}$ & $48-56$ & DBS $\alpha-G a l$ A & $\begin{array}{c}0(0 \mid 0) \\
{[0(0 \mid 0)]}\end{array}$ & p.R118C (1)\# & $\begin{array}{l}\text { Malavera [109], } \\
\text { 2020, Australia }\end{array}$ \\
\hline $\begin{array}{l}\text { Cryptogenic stroke } \\
{[114(75 \mid 39)]}\end{array}$ & $18-50$ & $\begin{array}{c}\text { DBS } \alpha-\text {-Gal A } \\
\text { (male), GLA } \\
\text { sequencing } \\
\text { (female) }\end{array}$ & $\begin{array}{c}3(2 \mid 1) \\
{[2.6(2.7 \mid 2.6)]}\end{array}$ & $\begin{array}{l}\text { p. F113I (1), intronic splice } \\
\text { variants (2) }\end{array}$ & $\begin{array}{l}\text { Afanasiev [113], } \\
\text { 2020, Israel }\end{array}$ \\
\hline
\end{tabular}

DBS: Dried blood spot; t: Excluding polymorphisms; \#: Polymorphisms (p.E66Q, p.D313Y, p.A143T, p.S126G and p.R118C). Mutation nomenclature followed the guidelines established by the Human Genome Variation Society (http://varnomen.hgvs.org/, accessed on 10 September 2021)

The highest frequency $(3.7 \%, 2 / 54)$ of GLA pathogenic variants was detected in screening performed by measuring $\alpha$-Gal A activity in individuals aged 22 to 55 years and with idiopathic strokes [108]. Moreover, screening by $\alpha$-Gal A activity and/or GLA sequencing of individuals with acute stroke or transient ischemic attack and aged 18 to 60 years demonstrated that three of 108 subjects $(2.7 \%)$ harbored GLA pathogenic variants [111]. However, no GLA pathogenic variant was found in 10 screenings of individuals with central neurological manifestations [103-106,109,110,114,115,118,119]. Furthermore, p.R227Q was the most frequent variant detected in three individuals in Turkey and France $[108,117]$, with this variant considered to be associated with the development of classical FD [42] and previously detected in one individual during the high-risk screening in individuals undergoing dialysis or kidney transplant in Turkey [53]. Additionally, IVS4+919G>A was detected in two Taiwanese individuals [120].

High-risk screenings of individuals with central nervous system manifestations were performed using a combination of $\alpha$-Gal A activity and GLA sequencing; $50 \%$ of these screenings detected no patient with GLA pathogenic variants. However, there were FD patients presenting only central neurological manifestations as FD manifestations, suggesting that central neurological manifestations are important for high-risk screening of FD, even if they are less frequent than other symptoms.

\section{High-Risk Screening to Detect Undiagnosed Patients with FD}

In Japan, the number of areas that perform NBS for FD is expanding; however, screening remains limited. Moreover, even with NBS, there is a possibility of false negatives, especially in females. Therefore, it is possible that there are many undiagnosed patients with FD. High-risk screening by GLA sequencing remains a challenge in Japan owing to cost and ethical issues. Thus, in the following sections, we discuss which individuals should be subjected to high-risk screening for FD.

\subsection{High-Risk Screening in Children and Adolescents}

As shown in our high-risk screening, $4.4 \%$ of individuals with peripheral neurological manifestations (e.g., limb pain and acroparesthesia) were diagnosed with FD in childhood and adolescence [13]. Therefore, during childhood and adolescence, and to detect classical FD patients and some heterozygous females, $\alpha$-Gal A activity should be measured for individuals with peripheral neurological manifestations, and GLA sequencing should be performed in individuals with impaired $\alpha$-Gal A activity.

Even for females without impaired $\alpha$-Gal A activity, measurement of $\alpha$-Gal A activity and/or GLA sequencing in male relatives with FD manifestations might lead to a diagnosis. In the absence of such a family history, GLA sequencing should be performed if the characteristics of the pain are more Fabry-like (e.g., exacerbated by increased body temperature or exercise) or if other initial FD manifestations are present (e.g., angioker- 
atoma, cornea verticillata, hypo-anhidrosis, gastrointestinal symptoms, proteinuria, and mulberries in urine).

\subsection{High-Risk Screening during Adolescence and in Older People}

During adolescence and at old ages, individuals with renal, cardiac, and central neurological manifestations should undergo high-risk screening for FD. Moreover, they should receive screening, diagnosis, and treatment as early as possible after developing initial manifestations in order to benefit from treatment [122,123]. For this purpose, initial manifestations of FD (e.g., proteinuria, albuminuria, and decreased eGFR as renal manifestations and LVH as a cardiac symptom) should be targeted for screening. Many individuals with these initial manifestations of FD could potentially be detected during medical check-ups. $\alpha$-Gal A activity measurement in DBS is considered an appropriate screening method for individuals with an initial manifestation of FD during a medical check-up, given the ease of use and utility of DBS.

\subsection{GLA Sequencing and Genetic Counseling for Families of Patients with FD}

In many high-risk screenings for FD, GLA sequencing was performed on family members of the proband, and many cases of FD have been diagnosed by family analysis [124].

There are no ethical issues associated with GLA sequencing for the diagnosis of family members that have already developed FD manifestations. However, if GLA sequencing is performed on family members that have not developed FD manifestations, genetic counseling should be provided to explain the significance of GLA sequencing and the potential impact of the results on family members.

One of the most difficult aspects of genetic counseling for FD is that heterozygous females can vary from asymptomatic to developing manifestations as severe as those observed in males with classical FD. Unfortunately, there is no way to predict whether a heterozygous female will develop the disease. A previous study reported that most heterozygous FD females $>68$ years of age develop LVH [125]. Therefore, it is necessary to follow up with heterozygous females that have not developed FD manifestations, given that they might eventually develop FD manifestations.

\section{Next-Generation Screening Method}

Lyso-Gb3 in the blood is used not only as a biomarker to evaluate the treatment effect [126] but also as a screening method, as described above. However, Lyso-Gb3 levels in the blood may not always be elevated in patients with late-onset FD or heterozygous female patients, making their use as a marker unsuitable for all FD patients. Currently, microRNAs are being studied as biomarkers and may be expected to be effective as new screening methods $[127,128]$.

\section{Conclusions}

FD manifestations vary depending on disease type and patient sex and age; therefore, it is important to establish a high-risk screening system that considers the age of the target population. The peripheral neurological manifestations observed in children and adolescents are specific to FD, making the probability of FD in these cases high. The renal, cardiac, and central neurological manifestations observed during adolescence and in older people are not specific for FD, and the frequency of FD is low, even among those with a high risk of FD. However, it is essential to establish and sustain a system capable of definitively diagnosing FD for physicians (especially pediatricians, cardiologists, nephrologists, and neurologists) in order to allow early diagnosis of FD.

Author Contributions: Conceptualization, T.S., J.K., K.S. and K.N.; writing-original draft preparation, T.S. and J.K.; writing-review and editing, J.K., K.S. and K.N.; visualization, T.S., J.K. and K.S.; supervision, J.K. and K.N.; project administration, J.K. and K.N.; funding acquisition, K.N. All authors have read and agreed to the published version of the manuscript. 
Funding: This study was supported in part by a Health and Labor Sciences Research Grant for Research on Rare and Intractable Diseases from the Ministry of Health, Labour and Welfare, Japan (grant number JPMH20FC1025); a Grant-in-Aid for Practical Research Project for Rare/Intractable Diseases from the Japan Agency for Medical Research and Development (AMED; grant numbers JP20ek0108482s0201, JP20gk0110050s0401); and a Grant-in-Aid for Scientific Research from the Ministry of Education, Culture, Sports, Science, and Technology, Japan (Japan Society for the Promotion of Science [JSPS] KAKENHI: grant number JP20K08207).

Institutional Review Board Statement: Not applicable.

Informed Consent Statement: Not applicable.

Data Availability Statement: Not applicable.

Acknowledgments: We are grateful to Fumiko Nozaki, Naomi Yano, Ayuko Tateishi, Emi Harakawa, Yasuyo Sakamoto, Hiroko Nasu, and Matsumi Harada for providing technical support related to this study.

Conflicts of Interest: The authors declare no conflict of interest.

\section{References}

1. Desnick, R.J.; Ioannou, Y.A.; Eng, C.M. Alpha-galactosidase A Deficiency: Fabry Disease. In The Metabolic and Molecular Bases of Inherited Disease; Scriver, C.R., Beaudet, A.L., Sly, W.S., Valle, D., Eds.; McGraw-Hill: New York, NY, USA, 2001; pp. $3733-3774$.

2. Sanchez-Nino, M.D.; Sanz, A.B.; Carrasco, S.; Saleem, M.A.; Mathieson, P.W.; Valdivielso, J.M.; Ruiz-Ortega, M.; Egido, J.; Ortiz, A. Globotriaosylsphingosine actions on human glomerular podocytes: Implications for Fabry nephropathy. Nephrol. Dial. Transplant. 2011, 26, 1797-1802. [CrossRef]

3. Echevarria, L.; Benistan, K.; Toussaint, A.; Dubourg, O.; Hagege, A.A.; Eladari, D.; Jabbour, F.; Beldjord, C.; De Mazancourt, P.; Germain, D.P. X-chromosome inactivation in female patients with Fabry disease. Clin. Genet. 2016, 89, 44-54. [CrossRef] [PubMed]

4. Beck, M.; Hughes, D.; Kampmann, C.; Larroque, S.; Mehta, A.; Pintos-Morell, G.; Ramaswami, U.; West, M.; Wijatyk, A.; Giugliani, R. Long-term effectiveness of agalsidase alfa enzyme replacement in Fabry disease: A Fabry Outcome Survey analysis. Mol. Genet. Metab. Rep. 2015, 3, 21-27. [CrossRef] [PubMed]

5. Veen, S.J.; Hollak, C.E.M.; Kuilenburg, A.B.P.; Langeveld, M. Developments in the treatment of Fabry disease. J. Inherit. Metab. Dis. 2020, 43, 908-921. [CrossRef]

6. $\quad$ Liguori, L.; Monticelli, M.; Allocca, M.; Hay Mele, B.; Lukas, J.; Cubellis, M.V.; Andreotti, G. Pharmacological Chaperones: A Therapeutic Approach for Diseases Caused by Destabilizing Missense Mutations. Int. J. Mol. Sci. 2020, 21, 489. [CrossRef] [PubMed]

7. Ringe, D.; Petsko, G.A. What are pharmacological chaperones and why are they interesting? J. Biol. 2009, 8, 80. [CrossRef] [PubMed]

8. McCafferty, E.H.; Scott, L.J. Migalastat: A Review in Fabry Disease. Drugs 2019, 79, 543-554. [CrossRef] [PubMed]

9. Cammisa, M.; Correra, A.; Andreotti, G.; Cubellis, M.V. Fabry_CEP: A tool to identify Fabry mutations responsive to pharmacological chaperones. Orphanet J. Rare Dis. 2013, 8, 111. [CrossRef]

10. Citro, V.; Cammisa, M.; Liguori, L.; Cimmaruta, C.; Lukas, J.; Cubellis, M.; Andreotti, G. The Large Phenotypic Spectrum of Fabry Disease Requires Graduated Diagnosis and Personalized Therapy: A Meta-Analysis Can Help to Differentiate Missense Mutations. Int. J. Mol. Sci. 2016, 17, 2010. [CrossRef]

11. Ferri, L.; Malesci, D.; Fioravanti, A.; Bagordo, G.; Filippini, A.; Ficcadenti, A.; Manna, R.; Antuzzi, D.; Verrecchia, E.; Donati, I.; et al. Functional and pharmacological evaluation of novel GLA variants in Fabry disease identifies six (two de novo ) causative mutations and two amenable variants to the chaperone DGJ. Clin. Chim. Acta 2018, 481, 25-33. [CrossRef]

12. Marchesoni, C.L.; Roa, N.; Pardal, A.M.; Neumann, P.; Cáceres, G.; Martínez, P.; Kisinovsky, I.; Bianchi, S.; Tarabuso, A.L.; Reisin, R.C. Misdiagnosis in Fabry disease. J. Pediatr. 2010, 156, 828-831. [CrossRef] [PubMed]

13. Yoshida, S.; Kido, J.; Sawada, T.; Momosaki, K.; Sugawara, K.; Matsumoto, S.; Endo, F.; Nakamura, K. Fabry disease screening in high-risk populations in Japan: A nationwide study. Orphanet J. Rare Dis. 2020, 15, 220. [CrossRef] [PubMed]

14. Sawada, T.; Kido, J.; Yoshida, S.; Sugawara, K.; Momosaki, K.; Inoue, T.; Tajima, G.; Sawada, H.; Mastumoto, S.; Endo, F.; et al. Newborn screening for Fabry disease in the western region of Japan. Mol. Genet. Metab. Rep. 2020, 22, 100562. [CrossRef] [PubMed]

15. Spada, M.; Pagliardini, S.; Yasuda, M.; Tukel, T.; Thiagarajan, G.; Sakuraba, H.; Ponzone, A.; Desnick, R.J. High Incidence of Later-Onset Fabry Disease Revealed by Newborn Screening*. Am. J. Hum. Genet. 2006, 79, 31-40. [CrossRef]

16. Liao, H.-C.; Chiang, C.-C.; Niu, D.-M.; Wang, C.-H.; Kao, S.-M.; Tsai, F.-J.; Huang, Y.-H.; Liu, H.-C.; Huang, C.-K.; Gao, H.-J.; et al. Detecting multiple lysosomal storage diseases by tandem mass spectrometry-A national newborn screening program in Taiwan. Clin. Chim. Acta 2014, 431, 80-86. [CrossRef] [PubMed] 
17. Lin, H.-Y.; Chong, K.-W.; Hsu, J.-H.; Yu, H.-C.; Shih, C.-C.; Huang, C.-H.; Lin, S.-J.; Chen, C.-H.; Chiang, C.-C.; Ho, H.-J.; et al. High Incidence of the Cardiac Variant of Fabry Disease Revealed by Newborn Screening in the Taiwan Chinese Population. Circ. Cardiovasc. Genet. 2009, 2, 450-456. [CrossRef]

18. Wasserstein, M.P.; Caggana, M.; Bailey, S.M.; Desnick, R.J.; Edelmann, L.; Estrella, L.; Holzman, I.; Kelly, N.R.; Kornreich, R.; Kupchik, S.G.; et al. The New York pilot newborn screening program for lysosomal storage diseases: Report of the First 65,000 Infants. Genet. Med. 2019, 21, 631-640. [CrossRef]

19. Felis, A.; Whitlow, M.; Kraus, A.; Warnock, D.G.; Wallace, E. Current and Investigational Therapeutics for Fabry Disease. Kidney Int. Rep. 2020, 5, 407-413. [CrossRef]

20. Mehta, A.; Ricci, R.; Widmer, U.; Dehout, F.; Garcia de Lorenzo, A.; Kampmann, C.; Linhart, A.; Sunder-Plassmann, G.; Ries, M.; Beck, M. Fabry disease defined: Baseline clinical manifestations of 366 patients in the Fabry Outcome Survey. Eur. J. Clin. Investig. 2004, 34, 236-242. [CrossRef]

21. Ganendiran, S.; Kramer, D.; Üçeyler, N.; Ganendiran, S.; Kramer, D.; Sommer, C. Characterization of Pain in Fabry Disease. Clin. J. Pain 2014, 30, 915-920.

22. Arends, M.; Wanner, C.; Hughes, D.; Mehta, A.; Oder, D.; Watkinson, O.T.; Elliott, P.M.; Linthorst, G.E.; Wijburg, F.A.; Biegstraaten, M.; et al. Characterization of Classical and Nonclassical Fabry Disease: A Multicenter Study. J. Am. Soc. Nephrol. 2017, 28, 1631-1641. [CrossRef]

23. Stiles, A.R.; Zhang, H.; Dai, J.; McCaw, P.; Beasley, J.; Rehder, C.; Koeberl, D.D.; McDonald, M.; Bali, D.S.; Young, S.P. A comprehensive testing algorithm for the diagnosis of Fabry disease in males and females. Mol. Genet. Metab. 2020, 130, 209-214. [CrossRef]

24. Linthorst, G.E.; Vedder, A.C.; Aerts, J.M.F.G.; Hollak, C.E.M. Screening for Fabry disease using whole blood spots fails to identify one-third of female carriers. Clin. Chim. Acta 2005, 353, 201-203. [CrossRef]

25. Moura, A.P.; Hammerschmidt, T.; Deon, M.; Giugliani, R.; Vargas, C.R. Investigation of correlation of urinary globotriaosylceramide (Gb3) levels with markers of renal function in patients with Fabry disease. Clin. Chim. Acta 2018, 478, 62-67. [CrossRef] [PubMed]

26. Üçeyler, N.; Böttger, J.; Henkel, L.; Langjahr, M.; Mayer, C.; Nordbeck, P.; Wanner, C.; Sommer, C. Detection of blood Gb3 deposits as a new tool for diagnosis and therapy monitoring in patients with classic Fabry disease. J. Intern. Med. 2018, 284, 427-438. [CrossRef]

27. Aerts, J.M.; Groener, J.E.; Kuiper, S.; Donker-Koopman, W.E.; Strijland, A.; Ottenhoff, R.; van Roomen, C.; Mirzaian, M.; Wijburg, F.A.; Linthorst, G.E.; et al. Elevated globotriaosylsphingosine is a hallmark of Fabry disease. Proc. Natl. Acad. Sci. USA 2008, 105, 2812-2817. [CrossRef] [PubMed]

28. Smid, B.E.; van der Tol, L.; Biegstraaten, M.; Linthorst, G.E.; Hollak, C.E.M.; Poorthuis, B.J.H.M. Plasma globotriaosylsphingosine in relation to phenotypes of Fabry disease. J. Med. Genet. 2015, 52, 262-268. [CrossRef]

29. Ishii, S.; Nakao, S.; Minamikawa-Tachino, R.; Desnick, R.J.; Fan, J.Q. Alternative splicing in the $\alpha$-galactosidase A gene: Increased exon inclusion results in the Fabry cardiac phenotype. Am. J. Hum. Genet. 2002, 70, 994-1002. [CrossRef] [PubMed]

30. Lenders, M.; Weidemann, F.; Kurschat, C.; Canaan-Kühl, S.; Duning, T.; Stypmann, J.; Schmitz, B.; Reiermann, S.; Krämer, J.; Blaschke, D.; et al. Alpha-Galactosidase A p.A143T, a non-Fabry disease-causing variant. Orphanet J. Rare Dis. 2016, 11, 54. [CrossRef]

31. Ferreira, S.; Ortiz, A.; Germain, D.P.; Viana-Baptista, M.; Caldeira-Gomes, A.; Camprecios, M.; Fenollar-Cortés, M.; GallegosVillalobos, Á.; Garcia, D.; García-Robles, J.A.; et al. The alpha-galactosidase A p.Arg118Cys variant does not cause a Fabry disease phenotype: Data from individual patients and family studies. Mol. Genet. Metab. 2015, 114, 248-258. [CrossRef]

32. Niemann, M.; Rolfs, A.; Giese, A.; Mascher, H.; Breunig, F.; Ertl, G.; Wanner, C.; Weidemann, F. Lyso-Gb3 Indicates that the Alpha-Galactosidase A Mutation D313Y is not Clinically Relevant for Fabry Disease. JIMD Rep. 2012, 7, 99-102.

33. Lukas, J.; Giese, A.-K.; Markoff, A.; Grittner, U.; Kolodny, E.; Mascher, H.; Lackner, K.J.; Meyer, W.; Wree, P.; Saviouk, V.; et al. Functional Characterisation of Alpha-Galactosidase A Mutations as a Basis for a New Classification System in Fabry Disease. PLoS Genet. 2013, 9, e1003632. [CrossRef]

34. Togawa, T.; Tsukimura, T.; Kodama, T.; Tanaka, T.; Kawashima, I.; Saito, S.; Ohno, K.; Fukushige, T.; Kanekura, T.; Satomura, A.; et al. Fabry disease: Biochemical, pathological and structural studies of the $\alpha$-galactosidase A with E66Q amino acid substitution. Mol. Genet. Metab. 2012, 105, 615-620. [CrossRef]

35. Peng, H.; Xu, X.; Zhang, L.; Zhang, X.; Peng, H.; Zheng, Y.; Luo, S.; Guo, H.; Xia, K.; Li, J.; et al. GLA variation p.E66Q identified as the genetic etiology of Fabry disease using exome sequencing. Gene 2016, 575, 363-367. [CrossRef]

36. Du Moulin, M.; Muschol, N. p.D313Y is more than just a polymorphism in Fabry disease. Clin. Genet. 2018, 93, 1258. [CrossRef]

37. Talbot, A.; Nicholls, K. Elevated Lyso-Gb3 Suggests the R118C GLA Mutation Is a Pathological Fabry Variant. JIMD Rep. 2019, $45,95$.

38. Burton, B.K.; Charrow, J.; Hoganson, G.E.; Waggoner, D.; Tinkle, B.; Braddock, S.R.; Schneider, M.; Grange, D.K.; Nash, C.; Shryock, H.; et al. Newborn Screening for Lysosomal Storage Disorders in Illinois: The Initial 15-Month Experience. J. Pediatr. 2017, 190, 130-135. [CrossRef]

39. Scott, C.R.; Elliott, S.; Buroker, N.; Thomas, L.I.; Keutzer, J.; Glass, M.; Gelb, M.H.; Turecek, F. Identification of Infants at Risk for Developing Fabry, Pompe, or Mucopolysaccharidosis-I from Newborn Blood Spots by Tandem Mass Spectrometry. J. Pediatr. 2013, 163, 498-503. [CrossRef] [PubMed] 
40. Nakao, S.; Takenaka, T.; Maeda, M.; Kodama, C.; Tanaka, A.; Tahara, M.; Yoshida, A.; Kuriyama, M.; Hayashibe, H.; Sakuraba, H.; et al. An Atypical Variant of Fabry's Disease in Men with Left Ventricular Hypertrophy. N. Engl. J. Med. 1995, 333, 288-293. [CrossRef] [PubMed]

41. Mitobe, S.; Togawa, T.; Tsukimura, T.; Kodama, T.; Tanaka, T.; Doi, K.; Noiri, E.; Akai, Y.; Saito, Y.; Yoshino, M.; et al. Mutant $\alpha$-galactosidase A with M296I does not cause elevation of the plasma globotriaosylsphingosine level. Mol. Genet. Metab. 2012, 107, 623-626. [CrossRef] [PubMed]

42. Germain, D.P.; Shabbeer, J.; Cotigny, S.; Desnick, R.J. Fabry Disease: Twenty Novel $\alpha$-Galactosidase A Mutations and GenotypePhenotype Correlations in Classical and Variant Phenotypes. Mol. Med. 2002, 8, 306-312. [CrossRef]

43. Sakuraba, H.; Tsukimura, T.; Togawa, T.; Tanaka, T.; Ohtsuka, T.; Sato, A.; Shiga, T.; Saito, S.; Ohno, K. Fabry disease in a Japanese population-molecular and biochemical characteristics. Mol. Genet. Metab. Rep. 2018, 17, 73-79. [CrossRef] [PubMed]

44. Sawada, T.; Kido, J.; Sugawara, K.; Matsumoto, S.; Takada, F.; Tsuboi, K.; Ohtake, A.; Endo, F.; Nakamura, K. Detection of novel Fabry disease-associated pathogenic variants in Japanese patients by newborn and high-risk screening. Mol. Genet. Genom. Med. 2020, 8, e1502. [CrossRef]

45. Yamamoto, S.; Nagasawa, T.; Sugimura, K.; Kanno, A.; Tatebe, S.; Aoki, T.; Sato, H.; Kozu, K.; Konno, R.; Nochioka, K.; et al. Clinical Diversity in Patients with Anderson-fabry Disease with the R301Q Mutation. Intern. Med. 2019, 58, 603-607. [CrossRef]

46. Utsumi, K.; Kase, R.; Takata, T.; Sakuraba, H.; Matsui, N.; Saito, H.; Nakamura, T.; Kawabe, M.; Iino, Y.; Katayama, Y. Fabry disease in patients receiving maintenance dialysis. Clin. Exp. Nephrol. 2000, 4, 49-51. [CrossRef]

47. Nakao, S.; Kodama, C.; Takenaka, T.; Tanaka, A.; Yasumoto, Y.; Yoshida, A.; Kanzaki, T.; Enriquez, A.L.D.; Eng, C.M.; Tanaka, H.; et al. Fabry disease: Detection of undiagnosed hemodialysis patients and identification of a "renal variant" phenotype1. Kidney Int. 2003, 64, 801-807. [CrossRef]

48. Veloso, V.S.P.; Ataides, T.L.; Canziani, M.E.F.; Veloso, M.P.; da Silva, N.A.; Barreto, D.V.; Pereira, E.R.S.; de Moura, L.A.R.; Barreto, F.C. A Novel Missense GLA Mutation (p.G35V) Detected in Hemodialysis Screening Leads to Severe Systemic Manifestations of Fabry Disease in Men and Women. Nephron 2018, 138, 147-156. [CrossRef]

49. Zizzo, C.; Testa, A.; Colomba, P.; Postorino, M.; Natale, G.; Pini, A.; Francofonte, D.; Cammarata, G.; Scalia, S.; Sciarrino, S.; et al. Systematic DNA Study for Fabry Disease in the End Stage Renal Disease Patients from a Southern Italy Area. Kidney Blood Press. Res. 2018, 43, 1344-1351. [CrossRef]

50. Kotanko, P. Results of a Nationwide Screening for Anderson-Fabry Disease among Dialysis Patients. J. Am. Soc. Nephrol. 2004, 15, 1323-1329. [CrossRef] [PubMed]

51. Frabasil, J.; Durand, C.; Sokn, S.; Gaggioli, D.; Carozza, P.; Carabajal, R.; Politei, J.; Schenone, A.B. Prevalence of Fabry disease in male dialysis patients: Argentinean screening study. JIMD Rep. 2019, 48, 45-52. [CrossRef]

52. Moiseev, S.; Fomin, V.; Savostyanov, K.; Pushkov, A.; Moiseev, A.; Svistunov, A.; Namazova-Baranova, L. The Prevalence and Clinical Features of Fabry Disease in Hemodialysis Patients: Russian Nationwide Fabry Dialysis Screening Program. Nephron 2019, 141, 249-255. [CrossRef] [PubMed]

53. Yalın, S.F.; Eren, N.; Sinangil, A.; Yilmaz, V.T.; Tatar, E.; Ucar, A.R.; Sevinc, M.; Can, Ö.; Gurkan, A.; Arik, N.; et al. Fabry Disease Prevalence in Renal Replacement Therapy in Turkey. Nephron 2019, 142, 26-33. [CrossRef] [PubMed]

54. Jahan, S.; Sarathchandran, S.; Akhter, S.; Goldblatt, J.; Stark, S.; Crawford, D.; Mallett, A.; Thomas, M. Prevalence of Fabry disease in dialysis patients: Western Australia Fabry disease screening study-The FoRWARD study. Orphanet J. Rare Dis. 2020, 15, 10. [CrossRef]

55. Veroux, M.; Monte, I.; Rodolico, M.; Corona, D.; Bella, R.; Basile, A.; Palmucci, S.; Pistorio, M.; Lanza, G.; De Pasquale, C.; et al. Screening for Fabry Disease in Kidney Transplant Recipients: Experience of a Multidisciplinary Team. Biomedicines 2020, 8, 396. [CrossRef]

56. Erdogmus, S.; Kutlay, S.; Kumru, G.; Ors Sendogan, D.; Erturk, S.; Keven, K.; Ceylaner, G.; Sengul, S. Fabry Disease Screening in Patients With Kidney Transplant: A Single-Center Study in Turkey. Exp. Clin. Transplant. 2020, 18, 444-449. [CrossRef]

57. Alhemyadi, S.A.; Elawad, M.; Fourtounas, K.; Abdrabbou, Z.; Alaraki, B.; Younis, S.; Nawaz, Z.; Alqurashi, S.; Mohamed, S. Screening for Fabry disease among 619 hemodialysis patients in Saudi Arabia. Saudi Med. J. 2020, 41, 813-818. [CrossRef]

58. Okur, I.; Ezgu, F.; Biberoglu, G.; Tumer, L.; Erten, Y.; Isitman, M.; Eminoglu, F.T.; Hasanoglu, A. Screening for Fabry disease in patients undergoing dialysis for chronic renal failure in Turkey: Identification of new case with novel mutation. Gene 2013, 527, 42-47. [CrossRef] [PubMed]

59. Vigneau, C.; Germain, D.P.; Larmet, D.; Jabbour, F.; Hourmant, M. Screening for Fabry disease in male patients with end-stage renal disease in western France. Néphrologie Thérapeutique 2021, 17, 180-184. [CrossRef]

60. Ichinose, M.; Nakayama, M.; Ohashi, T.; Utsunomiya, Y.; Kobayashi, M.; Eto, Y. Significance of screening for Fabry disease among male dialysis patients. Clin. Exp. Nephrol. 2005, 9, 228-232. [CrossRef] [PubMed]

61. Merta, M.; Reiterova, J.; Ledvinova, J.; Poupětová, H.; Dobrovolný, R.; Ryšavá, R.; Maixnerová, D.; Bultas, J.; Motáň, J.; Slivkova, J.; et al. A nationwide blood spot screening study for Fabry disease in the Czech Republic haemodialysis patient population. Nephrol. Dial. Transplant. 2007, 22, 179-186. [CrossRef]

62. Terryn, W.; Poppe, B.; Wuyts, B.; Claes, K.; Maes, B.; Verbeelen, D.; Vanholder, R.; De Boeck, K.; Lameire, N.; De Paepe, A.; et al. Two-tier approach for the detection of alpha-galactosidase A deficiency in a predominantly female haemodialysis population. Nephrol. Dial. Transplant. 2007, 23, 294-300. [CrossRef] 
63. Kim, J.-Y.; Hyun, Y.-Y.; Lee, J.-E.; Yoon, H.-R.; Kim, G.-H.; Yoo, H.-W.; Cho, S.-T.; Chun, N.-W.; Jeoung, B.-C.; Kim, H.-J.; et al. Serum Globotriaosylceramide Assay as a Screening Test for Fabry Disease in Patients with ESRD on Maintenance Dialysis in Korea. Korean J. Intern. Med. 2010, 25, 415. [CrossRef]

64. Gaspar, P.; Herrera, J.; Rodrigues, D.; Cerezo, S.; Delgado, R.; Andrade, C.F.; Forascepi, R.; Macias, J.; del Pino, M.D.; Prados, M.D.; et al. Frequency of Fabry disease in male and female haemodialysis patients in Spain. BMC Med. Genet. 2010, 11, 19. [CrossRef] [PubMed]

65. Nishino, T.; Obata, Y.; Furusu, A.; Hirose, M.; Shinzato, K.; Hattori, K.; Nakamura, K.; Matsumoto, T.; Endo, F.; Kohno, S. Identification of a Novel Mutation and Prevalence Study for Fabry Disease in Japanese Dialysis Patients. Ren. Fail. 2012, 34, 566-570. [CrossRef] [PubMed]

66. Doi, K.; Noiri, E.; Ishizu, T.; Negishi, K.; Suzuki, Y.; Hamasaki, Y.; Honda, K.; Fujita, T.; Tsukimura, T.; Togawa, T.; et al. High-throughput screening identified disease-causing mutants and functional variants of $\alpha$-galactosidase A gene in Japanese male hemodialysis patients. J. Hum. Genet. 2012, 57, 575-579. [CrossRef] [PubMed]

67. Maruyama, H.; Takata, T.; Tsubata, Y.; Tazawa, R.; Goto, K.; Tohyama, J.; Narita, I.; Yoshioka, H.; Ishii, S. Screening of Male Dialysis Patients for Fabry Disease by Plasma Globotriaosylsphingosine. Clin. J. Am. Soc. Nephrol. 2013, 8, 629-636. [CrossRef]

68. Rozenfeld, P.A.; Ceci, R.; Roa, N.; Kisinovsky, I. The Continuous Challenge of Diagnosing patients with Fabry disease in Argentina. J. Inborn Errors Metab. Screen. 2015, 3, 232640981561380. [CrossRef]

69. Saito, O.; Kusano, E.; Akimoto, T.; Asano, Y.; Kitagawa, T.; Suzuki, K.; Ishige, N.; Akiba, T.; Saito, A.; Ishimura, E.; et al. Prevalence of Fabry disease in dialysis patients: Japan Fabry disease screening study (J-FAST). Clin. Exp. Nephrol. 2016, 20, 284-293. [CrossRef] [PubMed]

70. Sayilar, E.I.; Ayar, Y.; Yavuz, M. Prevalence of Fabry disease among Turkish dialysis patients: Data from hemodialysis centers in Bursa province. Clin. Nephrol. 2016, 85, 165-172. [CrossRef]

71. Silva, C.A.B.; Barreto, F.C.; dos Reis, M.A.; Moura Junior, J.A.; Cruz, C.M.S. Targeted Screening of Fabry Disease in Male Hemodialysis Patients in Brazil Highlights Importance of Family Screening. Nephron 2016, 134, 221-230. [CrossRef]

72. Trachoo, O.; Jittorntam, P.; Pibalyart, S.; Kajanachumphol, S.; Suvachittanont, N.; Patputthipong, S.; Chuengsaman, P.; Nongnuch, A. Screening of Fabry disease in patients with end-stage renal disease of unknown etiology: The first Thailand study. J. Biomed. Res. 2017, 31, 17-24.

73. Yılmaz, M.; Uçar, S.K.; Aşçı, G.; Canda, E.; Tan, F.A.; Hoşcoşkun, C.; Çoker, M.; Töz, H. Preliminary Screening Results of Fabry Disease in Kidney Transplantation Patients: A Single-Center Study. Transplant. Proc. 2017, 49, 420-424. [CrossRef] [PubMed]

74. Andrade, J.; Waters, P.J.; Singh, R.S.; Levin, A.; Toh, B.-C.; Vallance, H.D.; Sirrs, S. Screening for Fabry Disease in Patients with Chronic Kidney Disease: Limitations of Plasma $\alpha$-Galactosidase Assay as a Screening Test. Clin. J. Am. Soc. Nephrol. 2008, 3, 139-145. [CrossRef] [PubMed]

75. Turkmen, K.; Guclu, A.; Sahin, G.; Kocyigit, I.; Demirtas, L.; Erdur, F.M.; Sengül, E.; Ozkan, O.; Emre, H.; Turgut, F.; et al. The Prevalence of Fabry Disease in Patients with Chronic Kidney Disease in Turkey: The TURKFAB Study. Kidney Blood Press. Res. 2016, 41, 1016-1024. [CrossRef]

76. Yeniçerioğlu, Y.; Akdam, H.; Dursun, B.; Alp, A.; Sağlam Eyiler, F.; Akın, D.; Gün, Y.; Hüddam, B.; Batmazoğlu, M.; Gibyeli Genek, D.; et al. Screening Fabry's disease in chronic kidney disease patients not on dialysis: A multicenter study. Ren. Fail. 2017, 39, 104-111. [CrossRef] [PubMed]

77. Lin, C.-J.; Chien, Y.-H.; Lai, T.-S.; Shih, H.-M.; Chen, Y.-C.; Pan, C.-F.; Chen, H.-H.; Hwu, W.-L.; Wu, C.-J. Results of Fabry Disease Screening in Male Pre-End Stage Renal Disease Patients with Unknown Etiology Found Through the Platform of a Chronic Kidney Disease Education Program in a Northern Taiwan Medical Center. Kidney Blood Press. Res. 2018, 43, 1636-1645. [CrossRef]

78. Auray-Blais, C.; Lavoie, P.; Abaoui, M.; Côté, A.-M.; Boutin, M.; Akbari, A.; Levin, A.; Mac-Way, F.; TR Clarke, J. High-risk screening for Fabry disease in a Canadian cohort of chronic kidney disease patients. Clin. Chim. Acta 2020, 501, 234-240. [CrossRef]

79. Reynolds, T.M.; Tylee, K.L.; Booth, K.L.; Wierzbicki, A.S. Identification of patients with Fabry disease using routine pathology results: PATHFINDER (eGFR) study. Int. J. Clin. Pract. 2021, 75.

80. Sachdev, B.; Takenaka, T.; Teraguchi, H.; Tei, C.; Lee, P.; McKenna, W.J.; Elliott, P.M. Prevalence of Anderson-Fabry Disease in Male Patients With Late Onset Hypertrophic Cardiomyopathy. Circulation 2002, 105, 1407-1411. [CrossRef]

81. Fuller, M.; Perry, R.; Saiedi, M.; Fletcher, J.M.; Selvanayagam, J.B. Mono-symptomatic Fabry disease in a population with mild-to-moderate left ventricular hypertrophy. Mol. Genet. Metab. Rep. 2020, 25, 100697. [CrossRef]

82. Fan, Y.; Chan, T.-N.; Chow, J.T.Y.; Kam, K.K.H.; Chi, W.-K.; Chan, J.Y.S.; Fung, E.; Tong, M.M.P.; Wong, J.K.T.; Choi, P.C.L.; et al. High Prevalence of Late-Onset Fabry Cardiomyopathy in a Cohort of 499 Non-Selective Patients with Left Ventricular Hypertrophy: The Asian Fabry Cardiomyopathy High-Risk Screening Study (ASIAN-FAME). J. Clin. Med. 2021, 10, 2160. [CrossRef] [PubMed]

83. Monserrat, L.; Gimeno-Blanes, J.R.; Marín, F.; Hermida-Prieto, M.; García-Honrubia, A.; Pérez, I.; Fernández, X.; de Nicolas, R.; de la Morena, G.; Payá, E.; et al. Prevalence of Fabry Disease in a Cohort of 508 Unrelated Patients With Hypertrophic Cardiomyopathy. J. Am. Coll. Cardiol. 2007, 50, 2399-2403. [CrossRef] [PubMed]

84. Hagege, A.A.; Caudron, E.; Damy, T.; Roudaut, R.; Millaire, A.; Etchecopar-Chevreuil, C.; Tran, T.-C.; Jabbour, F.; Boucly, C.; Prognon, P.; et al. Screening patients with hypertrophic cardiomyopathy for Fabry disease using a filter-paper test: The FOCUS study. Heart 2011, 97, 131-136. [CrossRef] 
85. Mawatari, K.; Yasukawa, H.; Oba, T.; Nagata, T.; Togawa, T.; Tsukimura, T.; Kyogoku, S.; Ohshima, H.; Minami, T.; Sugi, Y.; et al. Screening for Fabry disease in patients with left ventricular hypertrophy. Int. J. Cardiol. 2013, 167, 1059-1061. [CrossRef] [PubMed]

86. Terryn, W.; Deschoenmakere, G.; De Keyser, J.; Meersseman, W.; Van Biesen, W.; Wuyts, B.; Hemelsoet, D.; Pascale, H.; De Backer, J.; De Paepe, A.; et al. Prevalence of Fabry disease in a predominantly hypertensive population with left ventricular hypertrophy. Int. J. Cardiol. 2013, 167, 2555-2560. [CrossRef]

87. Palecek, T.; Honzikova, J.; Poupetova, H.; Vlaskova, H.; Kuchynka, P.; Golan, L.; Magage, S.; Linhart, A. Prevalence of Fabry disease in male patients with unexplained left ventricular hypertrophy in primary cardiology practice: Prospective Fabry cardiomyopathy screening study (FACSS). J. Inherit. Metab. Dis. 2014, 37, 455-460. [CrossRef]

88. Baptista, A.; Magalhães, P.; Leão, S.; Carvalho, S.; Mateus, P.; Moreira, I. Screening for Fabry Disease in Left Ventricular Hypertrophy: Documentation of a Novel Mutation. Arq. Bras. Cardiol. 2015, 105, 139-144. [CrossRef]

89. Gaggl, M.; Lajic, N.; Heinze, G.; Voigtländer, T.; Sunder-Plassmann, R.; Paschke, E.; Fauler, G.; Sunder-Plassmann, G.; Mundigler, G. Screening for Fabry Disease by Urinary Globotriaosylceramide Isoforms Measurement in Patients with Left Ventricular Hypertrophy. Int. J. Med. Sci. 2016, 13, 340-346. [CrossRef]

90. Seo, J.; Kim, M.; Hong, G.-R.; Kim, D.-S.; Son, J.-W.; Cho, I.J.; Shim, C.Y.; Chang, H.-J.; Ha, J.-W.; Chung, N. Fabry disease in patients with hypertrophic cardiomyopathy: A practical approach to diagnosis. J. Hum. Genet. 2016, 61, 775-780. [CrossRef]

91. Elliott, P.; Baker, R.; Pasquale, F.; Quarta, G.; Ebrahim, H.; Mehta, A.B.; Hughes, D.A. Prevalence of Anderson-Fabry disease in patients with hypertrophic cardiomyopathy: The European Anderson-Fabry Disease Survey. Heart 2011, 97, 1957-1960. [CrossRef]

92. Kubo, T.; Ochi, Y.; Baba, Y.; Hirota, T.; Tanioka, K.; Yamasaki, N.; Yoshimitsu, M.; Higuchi, K.; Takenaka, T.; Nakajima, K.; et al. Prevalence and clinical features of Fabry disease in Japanese male patients with diagnosis of hypertrophic cardiomyopathy. $J$. Cardiol. 2017, 69, 302-307. [CrossRef] [PubMed]

93. Maron, M.S.; Xin, W.; Sims, K.B.; Butler, R.; Haas, T.S.; Rowin, E.J.; Desnick, R.J.; Maron, B.J. Identification of Fabry Disease in a Tertiary Referral Cohort of Patients with Hypertrophic Cardiomyopathy. Am. J. Med. 2018, 131, 200.e1-200.e8. [CrossRef]

94. Kim, W.-S.; Kim, H.S.; Shin, J.; Park, J.C.; Yoo, H.-W.; Takenaka, T.; Tei, C. Prevalence of Fabry Disease in Korean Men with Left Ventricular Hypertrophy. J. Korean Med. Sci. 2019, 34, e63. [CrossRef] [PubMed]

95. Yamashita, S.; Saotome, M.; Satoh, H.; Kajihara, J.; Mochizuki, Y.; Mizuno, K.; Nobuhara, M.; Miyajima, K.; Kumazawa, A.; Tominaga, H.; et al. Plasma Globotriaosylsphingosine Level as a Primary Screening Target for Fabry Disease in Patients With Left Ventricular Hypertrophy. Circ. J. 2019, 83, 1901-1907. [CrossRef]

96. Barman, H.A.; Ikitimur, B.; Avci, B.K.; Durmaz, E.; Atici, A.; Aslan, S.; Ceylaner, S.; Karpuz, H. The Prevalence of Fabry Disease Among Turkish Patients with Non-obstructive Hypertrophic Cardiomyopathy: Insights from a Screening Study. Balk. Med. J. 2019, 36, 354. [CrossRef] [PubMed]

97. Sadasivan, C.; Chow, J.T.Y.; Sheng, B.; Chan, D.K.H.; Fan, Y.; Choi, P.C.L.; Wong, J.K.T.; Tong, M.M.B.; Chan, T.-N.; Fung, E.; et al. Screening for Fabry Disease in patients with unexplained left ventricular hypertrophy. PLoS ONE 2020, 15, e0239675. [CrossRef] [PubMed]

98. Barman, H.A. Ratio of Fabry Disease in patients with idiopathic Left Ventricular Hypertrophy: A single center study from Turkey. Anatol. J. Cardiol. 2019, 23, 86-98. [CrossRef] [PubMed]

99. Schiffmann, R.; Swift, C.; McNeill, N.; Benjamin, E.R.; Castelli, J.P.; Barth, J.; Sweetman, L.; Wang, X.; Wu, X. Low frequency of Fabry disease in patients with common heart disease. Genet. Med. 2018, 20, 754-759. [CrossRef]

100. López-Sainz, Á.; Climent, V.; Ripoll-Vera, T.; Espinosa, M.A.; Barriales-Villa, R.; Navarro, M.; Limeres, J.; Domingo, D.; Kasper, D.C.; Garcia-Pavia, P. Negative screening of Fabry disease in patients with conduction disorders requiring a pacemaker. Orphanet J. Rare Dis. 2019, 14, 170. [CrossRef]

101. Azevedo, O.; Marques, N.; Craveiro, N.; Pereira, A.R.; Antunes, H.; Reis, L.; Guerreiro, R.A.; Pontes dos Santos, R.; MiltenbergerMiltenyi, G.; Sousa, N.; et al. Screening for Fabry disease in patients with left ventricular noncompaction. Rev. Port. Cardiol. 2019, 38, 709-716. [CrossRef]

102. Germain, D.P.; Brand, E.; Burlina, A.; Cecchi, F.; Garman, S.C.; Kempf, J.; Laney, D.A.; Linhart, A.; Maródi, L.; Nicholls, K.; et al. Phenotypic characteristics of the p.Asn215Ser (p.N215S) GLA mutation in male and female patients with Fabry disease: A multicenter Fabry Registry study. Mol. Genet. Genom. Med. 2018, 6, 492-503. [CrossRef] [PubMed]

103. Brouns, R.; Sheorajpanday, R.; Braxel, E.; Eyskens, F.; Baker, R.; Hughes, D.; Mehta, A.; Timmerman, T.; Vincent, M.-F.; De Deyn, P.P. Middelheim Fabry Study (MiFaS): A retrospective Belgian study on the prevalence of Fabry disease in young patients with cryptogenic stroke. Clin. Neurol. Neurosurg. 2007, 109, 479-484. [CrossRef] [PubMed]

104. Wozniak, M.A.; Kittner, S.J.; Tuhrim, S.; Cole, J.W.; Stern, B.; Dobbins, M.; Grace, M.E.; Nazarenko, I.; Dobrovolny, R.; McDade, E.; et al. Frequency of Unrecognized Fabry Disease Among Young European-American and African-American Men With First Ischemic Stroke. Stroke 2010, 41, 78-81. [CrossRef] [PubMed]

105. Kinoshita, N.; Hosomi, N.; Matsushima, H.; Nakamori, M.; Yagita, Y.; Yamawaki, T.; Torii, T.; Kitamura, T.; Sueda, Y.; Shimomura, R.; et al. Screening for Fabry Disease in Japanese Patients with Young-Onset Stroke by Measuring $\alpha$-Galactosidase A and Globotriaosylsphingosine. J. Stroke Cerebrovasc. Dis. 2018, 27, 3563-3569. [CrossRef] [PubMed]

106. Sarikaya, H.; Yilmaz, M.; Michael, N.; Miserez, A.R.; Steinmann, B.; Baumgartner, R.W. Zurich Fabry study-Prevalence of Fabry disease in young patients with first cryptogenic ischaemic stroke or TIA. Eur. J. Neurol. 2012, 19, 1421-1426. [CrossRef] [PubMed] 
107. Nagamatsu, K.; Sekijima, Y.; Nakamura, K.; Nakamura, K.; Hattori, K.; Ota, M.; Shimizu, Y.; Endo, F.; Ikeda, S. Prevalence of Fabry disease and GLA c.196G>C variant in Japanese stroke patients. J. Hum. Genet. 2017, 62, 665-670. [CrossRef]

108. Gündoğdu, A.A.; Kotan, D.; Alemdar, M. The Frequency of Fabry Disease among Young Cryptogenic Stroke Patients in the City of Sakarya. J. Stroke Cerebrovasc. Dis. 2017, 26, 1334-1340. [CrossRef]

109. Malavera, A.; Cadilhac, D.A.; Thijs, V.; Lim, J.Y.; Grabsch, B.; Breen, S.; Jan, S.; Anderson, C.S. Screening for Fabry Disease in Young Strokes in the Australian Stroke Clinical Registry (AuSCR). Front. Neurol. 2020, 11, 596420. [CrossRef]

110. Brouns, R.; Thijs, V.; Eyskens, F.; Van den Broeck, M.; Belachew, S.; Van Broeckhoven, C.; Redondo, P.; Hemelsoet, D.; Fumal, A.; Jeangette, S.; et al. Belgian Fabry study: Prevalence of Fabry disease in a cohort of 1000 young patients with cerebrovascular disease. Stroke 2010, 41, 863-868. [CrossRef] [PubMed]

111. Romani, I.; Borsini, W.; Nencini, P.; Morrone, A.; Ferri, L.; Frusconi, S.; Donadio, V.A.; Liguori, R.; Donati, M.A.; Falconi, S.; et al. De novo Diagnosis of Fabry Disease among Italian Adults with Acute Ischemic Stroke or Transient Ischemic Attack. J. Stroke Cerebrovasc. Dis. 2015, 24, 2588-2595. [CrossRef] [PubMed]

112. Reisin, R.C.; Mazziotti, J.; Cejas, L.L.; Zinnerman, A.; Bonardo, P.; Pardal, M.F.; Martínez, A.; Riccio, P.; Ameriso, S.; Bendersky, E.; et al. Prevalence of Fabry Disease in Young Patients with Stroke in Argentina. J. Stroke Cerebrovasc. Dis. 2018, $27,575-582$. [CrossRef] [PubMed]

113. Afanasiev, S.; Telman, G.; Hadad, R.; Altarescu, G. Fabry Disease in Young Ischemic Stroke Patients in Northern Israel. J. Stroke Cerebrovasc. Dis. 2020, 29, 105398. [CrossRef]

114. Baptista, M.V.; Ferreira, S.; Pinho-e-Melo, T.; Carvalho, M.; Cruz, V.T.; Carmona, C.; Silva, F.A.; Tuna, A.; Rodrigues, M.; Ferreira, C.; et al. Mutations of the GLA Gene in Young Patients With Stroke. Stroke 2010, 41, 431-436. [CrossRef]

115. Marquardt, L.; Baker, R.; Segal, H.; Burgess, A.I.; Poole, D.; Hughes, D.A.; Rothwell, P.M. Fabry disease in unselected patients with TIA or stroke: Population-based study. Eur. J. Neurol. 2012, 19, 1427-1432. [CrossRef]

116. Rolfs, A.; Fazekas, F.; Grittner, U.; Dichgans, M.; Martus, P.; Holzhausen, M.; Böttcher, T.; Heuschmann, P.U.; Tatlisumak, T.; Tanislav, C.; et al. Acute Cerebrovascular Disease in the Young. Stroke 2013, 44, 340-349. [CrossRef] [PubMed]

117. Fancellu, L.; Borsini, W.; Romani, I.; Pirisi, A.; Deiana, G.A.; Sechi, E.; Doneddu, P.E.; Rassu, A.L.; Demurtas, R.; Scarabotto, A.; et al. Exploratory screening for Fabry's disease in young adults with cerebrovascular disorders in northern Sardinia. BMC Neurol. 2015, 15, 256. [CrossRef]

118. Kilarski, L.L.; Rutten-Jacobs, L.C.A.; Bevan, S.; Baker, R.; Hassan, A.; Hughes, D.A.; Markus, H.S. Prevalence of CADASIL and Fabry Disease in a Cohort of MRI Defined Younger Onset Lacunar Stroke. PLoS ONE 2015, 10, e0136352. [CrossRef]

119. Lanthier, S.; Saposnik, G.; Lebovic, G.; Pope, K.; Selchen, D.; Moore, D.F.; Selchen, D.; Boulanger, J.-M.; Buck, B.; Butcher, K.; et al. Prevalence of Fabry Disease and Outcomes in Young Canadian Patients With Cryptogenic Ischemic Cerebrovascular Events. Stroke 2017, 48, 1766-1772. [CrossRef]

120. Lee, T.-H.; Yang, J.-T.; Lee, J.-D.; Chang, K.-C.; Peng, T.-I.; Chang, T.-Y.; Huang, K.-L.; Liu, C.-H.; Ryu, S.-J.; Burlina, A.P. Genomic screening of Fabry disease in young stroke patients: The Taiwan experience and a review of the literature. Eur. J. Neurol. 2019, 26, 553-555. [CrossRef]

121. Dubuc, V.; Moore, D.F.; Gioia, L.C.; Saposnik, G.; Selchen, D.; Lanthier, S. Prevalence of Fabry Disease in Young Patients with Cryptogenic Ischemic Stroke. J. Stroke Cerebrovasc. Dis. 2013, 22, 1288-1292. [CrossRef]

122. Germain, D.P.; Elliott, P.M.; Falissard, B.; Fomin, V.V.; Hilz, M.J.; Jovanovic, A.; Kantola, I.; Linhart, A.; Mignani, R.; Namdar, M.; et al. The effect of enzyme replacement therapy on clinical outcomes in male patients with Fabry disease: A systematic literature review by a European panel of experts. Mol. Genet. Metab. Rep. 2019, 19, 100454. [CrossRef] [PubMed]

123. Germain, D.P.; Arad, M.; Burlina, A.; Elliott, P.M.; Falissard, B.; Feldt-Rasmussen, U.; Hilz, M.J.; Hughes, D.A.; Ortiz, A.; Wanner, C.; et al. The effect of enzyme replacement therapy on clinical outcomes in female patients with Fabry disease-A systematic literature review by a European panel of experts. Mol. Genet. Metab. 2019, 126, 224-235. [CrossRef] [PubMed]

124. Germain, D.P.; Moiseev, S.; Suárez-Obando, F.; Al Ismaili, F.; Al Khawaja, H.; Altarescu, G.; Barreto, F.C.; Haddoum, F.; Hadipour, F; Maksimova, I.; et al. The benefits and challenges of family genetic testing in rare genetic diseases-lessons from Fabry disease. Mol. Genet. Genomic Med. 2021, 9, 1666. [CrossRef] [PubMed]

125. Kobayashi, M.; Ohashi, T.; Sakuma, M.; Ida, H.; Eto, Y. Clinical manifestations and natural history of Japanese heterozygous females with Fabry disease. J. Inherit. Metab. Dis. 2008, 31, 483-487. [CrossRef]

126. Rombach, S.M.; Aerts, J.M.F.G.; Poorthuis, B.J.H.M.; Groener, J.E.M.; Donker-Koopman, W.; Hendriks, E.; Mirzaian, M.; Kuiper, S.; Wijburg, F.A.; Hollak, C.E.M.; et al. Long-Term Effect of Antibodies against Infused Alpha-Galactosidase A in Fabry Disease on Plasma and Urinary (lyso)Gb3 Reduction and Treatment Outcome. PLoS ONE 2012, 7, e47805. [CrossRef]

127. Xiao, K.; Lu, D.; Hoepfner, J.; Santer, L.; Gupta, S.; Pfanne, A.; Thum, S.; Lenders, M.; Brand, E.; Nordbeck, P.; et al. Circulating microRNAs in Fabry Disease. Sci. Rep. 2019, 9, 15277. [CrossRef]

128. Cammarata, G.; Scalia, S.; Colomba, P.; Zizzo, C.; Pisani, A.; Riccio, E.; Montalbano, M.; Alessandro, R.; Giordano, A.; Duro, G. A pilot study of circulating microRNAs as potential biomarkers of Fabry disease. Oncotarget 2018, 9, 27333-27345. [CrossRef] 